\title{
Efficient Explicit Formulae for Genus 2 Hyperelliptic Curves over Prime Fields and Their Implementations
}

\author{
Xinxin Fan and Guang Gong \\ Department of Electrical and Computer Engineering \\ University of Waterloo \\ Waterloo, Ontario, N2L 3G1, Canada \\ x5fan@engmail.uwaterloo.ca, G.Gong@ece.uwaterloo.ca
}

\begin{abstract}
We analyze all the cases and propose the corresponding explicit formulae for computing $2 D_{1}+D_{2}$ in one step from given divisor classes $D_{1}$ and $D_{2}$ on genus 2 hyperelliptic curves defined over prime fields. Compared with naive method, the improved formula can save two field multiplications and one field squaring each time when the arithmetic is performed in the most frequent case. Furthermore, we present a variant which trades one field inversion for fourteen field multiplications and two field squarings by using Montgomery's trick to combine the two inversions. Experimental results show that our algorithms can save up to $13 \%$ of the time to perform a scalar multiplication on a general genus 2 hyperelliptic curve over a prime field, when compared with the best known general methods.
\end{abstract}

Keywords: Genus 2 hyperelliptic curves, explicit formulae, Cantor's algorithm, Harley's variant, efficient implementation.

\section{Introduction}

In 1988, Koblitz proposed for the first time to use the Jacobian of a hyperelliptic curve (HEC) defined over a finite field to implement cryptographic protocols based on the difficulty of the discrete logarithm problem [14. During the past few years, hyperelliptic curve cryptosystems (HECC) have become increasing popular for use in practice to provide an alternative to the widely used elliptic curve cryptosystems (ECC) because of much shorter operand length than that of ECC. Moreover, recent research has also shown that HECC are well suited for various software and hardware platforms and their performance is compatible to that of ECC [142122].

The most important and expensive operation in ECC and HECC is the scalar multiplication by an integer $k$, i.e., computing a scalar multiple $k P$ of a point $P$ on the points group or $k D$ of a divisor class $D$ on the Jacobian, where $k$ might be 160 bits or more. Various techniques for efficiently computing the scalar multiplication have been proposed 213. For general elliptic curves, Eisenträger et al. proposed a very elegant method for accelerating the scalar multiplication [10].

C. Adams, A. Miri, and M. Wiener (Eds.): SAC 2007, LNCS 4876, pp. 155-172, 2007.

(C) Springer-Verlag Berlin Heidelberg 2007 
Their improvements are based on the efficient computation of $2 P+Q$ in one step from given points $P$ and $Q$ on an elliptic curve. Since the point doubling is slightly more expensive than the point addition in the group operations of ECC, it is more efficient to calculate $2 P+Q$ as $P+(P+Q)$ than first doubling $P$ and then adding $Q$. This trick can save one field multiplication each time the certain sequence of operations occurs. Furthermore, their method finds applications to simultaneous multiple scalar multiplication, the Elliptic Curve Method of factorization, as well as the computation of the Weil and Tate pairings [10]. In the rest of this paper $I$ represents a field inversion, $M$ a field multiplication, and $S$ a field squaring.

This work generalizes Eisenträger et al.'s idea to genus 2 HECs over prime fields where the group doubling costs two more field squarings than the group addition [16]. We analyze all the possible cases during the computation procedure of $2 D_{1}+D_{2}$ from given divisor classes $D_{1}$ and $D_{2}$ on a genus $2 \mathrm{HEC}$ over $\mathbb{F}_{p}$. For the most frequent case, we propose a basic algorithm and its variant which cost $2 I+42 M+5 S$ and $1 I+56 M+7 S$, respectively, to compute $2 D_{1}+D_{2}$ in one step. Compared to the naive method using two separate group additions, our basic algorithm can save $2 M+1 S$. In the variant, which is faster whenever one inversion is more expensive than about sixteen field multiplications, Montgomery's trick 8 is employed to combine the two inversions in the basic algorithm. Furthermore, we implement the proposed algorithms on a Pentium processor to verify the correctness and test the performance of our new explicit formulae. For genus 2 HECs over binary fields, the fastest doubling formula, which requires only half the time of an addition, has been obtained by Lange and Stevens for a special family of curves [17. We note that the Eisenträger et al.'s trick can not be applied to optimize the computation of $2 D_{1}+D_{2}$ for the special family of genus 2 curves over binary fields when the group doubling is more efficient than the group addition.

The rest of this paper is organized as follows: Section 2 gives a short introduction to the mathematical background of genus 2 HECs over prime fields. Section 3 makes a thorough case study for the computation of $2 D_{1}+D_{2}$, presents the corresponding explicit formulae and analyzes the cost of the NAF scalar multiplication. Section 4 gives the experimental results of our newly derived explicit formulae. Finally, Section 5 ends this contribution.

\section{Mathematical Background on Genus 2 Hyperelliptic Curves over Prime Fields}

In this section, we present a brief introduction to the theory of genus 2 hyperelliptic curves over prime fields, restricting attention to the material which is relevant to this work. For more details, the reader is referred to [36 15 18].

Let $\mathbb{F}_{q}$ be a finite field of characteristic $p \neq 2, q=p^{n}$, and let $\overline{\mathbb{F}}_{q}$ denote the algebraic closure of $\mathbb{F}_{q}$. Let $\mathbb{F}_{q}(C) / \mathbb{F}_{q}$ be a quadratic function field defined via an equation

$$
C: Y^{2}=F(X)
$$


where $F(X)=X^{5}+f_{4} X^{4}+f_{3} X^{3}+f_{2} X^{2}+f_{1} X+f_{0} \in \mathbb{F}_{q}[X]$ is a monic and square-free polynomial of degree 5 . The curve $C / \mathbb{F}_{q}$ associated with this function field is called a hyperelliptic curve of genus 2 defined over $\mathbb{F}_{q}$. For our purpose it is enough to consider a point $P$ as an ordered pair $P=(x, y) \in \overline{\mathbb{F}}_{q}^{2}$ which satisfies $y^{2}=F(x)$. Besides these tuples there is one point $P_{\infty}$ at infinity. The inverse of $P$ is defined as $-P=(x,-y)$. We call a point $P$ that satisfies $P=-P$ a ramification point. Note that for $p \neq 5$ the transform $X \rightarrow X-f_{4} / 5$ makes the coefficient of $X^{4}$ in $F(X)$ zero.

The divisor class group $\mathcal{J}_{C}\left(\mathbb{F}_{q}\right)$ of $C$ forms a finite Abelian group and therefore we can construct cryptosystems whose security is based on the difficulty of the discrete logarithm problem on the Jacobian of $C$. Each element of the Jacobian can be represented uniquely by a so-called reduced divisor [6]. Mumford [19] showed that a reduced divisor can be represented by means of two polynomials $U(X), V(X) \in \mathbb{F}_{q}[X]$, where $U(X)$ and $V(X)$ satisfy the following three conditions: (i) $U(X)$ is monic, (ii) $\operatorname{deg} V(X)<\operatorname{deg} U(X) \leq 2$, and (iii) $U(X) \mid V(X)^{2}-F(X)$. In the remainder of this paper, we will use the notation $[U, V]$ for the divisor class represented by $U(X)$ and $V(X)$. For a genus 2 HEC, we have commonly $[U, V]=\left[X^{2}+u_{1} X+u_{0}, v_{1} X+v_{0}\right]$.

Cantor's algorithm [6] describes how to perform the group addition of two divisor classes in Mumford's representation. We review Cantor's algorithm for genus 2 HECs over prime fields in the following Algorithm 1. Cantor's algorithm only involves polynomial arithmetic over the finite field in which the divisor class group is defined. However, there are some redundant computations of the polynomial's coefficients in this classical algorithm. In order to simplify Cantor's algorithm, Harley proposed the first explicit formulae for a group addition and a group doubling of divisor classes on $\mathcal{J}_{C}\left(\mathbb{F}_{q}\right)$ in 2000. In [1], Gaudry and Harley significantly reduced the computational complexity of the group operations by distinguishing different cases according to the properties of the input divisor classes. They presented a very efficient algorithm, which uses many modern polynomial computation techniques such as Chinese remainder theorem, Newton's iteration, and Karatsuba's multiplication. Algorithm 2 gives a high level description of Harley's variant for adding two reduced divisor classes in the most frequent case for genus 2 HECs over prime fields. The most frequent cases mean that for the addition the inputs are two co-prime polynomials of degree 2 , which occur with the overwhelming probability [20], and the remainder cases are called exceptional cases. For more details about Cantor's algorithm and Harley's variant, the reader is referred to 3 16]22].

\section{Efficient Algorithms for Computing $2 D_{1}+D_{2}$}

In this section we adapt the idea of [10] to genus 2 HECs over prime fields. We obtain $D_{3}=2 D_{1}+D_{2}$ by the following two steps: we first compute $D^{\prime}=$ $\left[U^{\prime}, V^{\prime}\right]=D_{1}+D_{2}$ and omit the computation of the coefficients of $V^{\prime}$ because $V^{\prime}$ will not be used in the next phase. And then, we find $D_{3}=D^{\prime}+D_{1}$. Hence, we use two group additions to form $2 D_{1}+D_{2}$ instead of a group addition and 

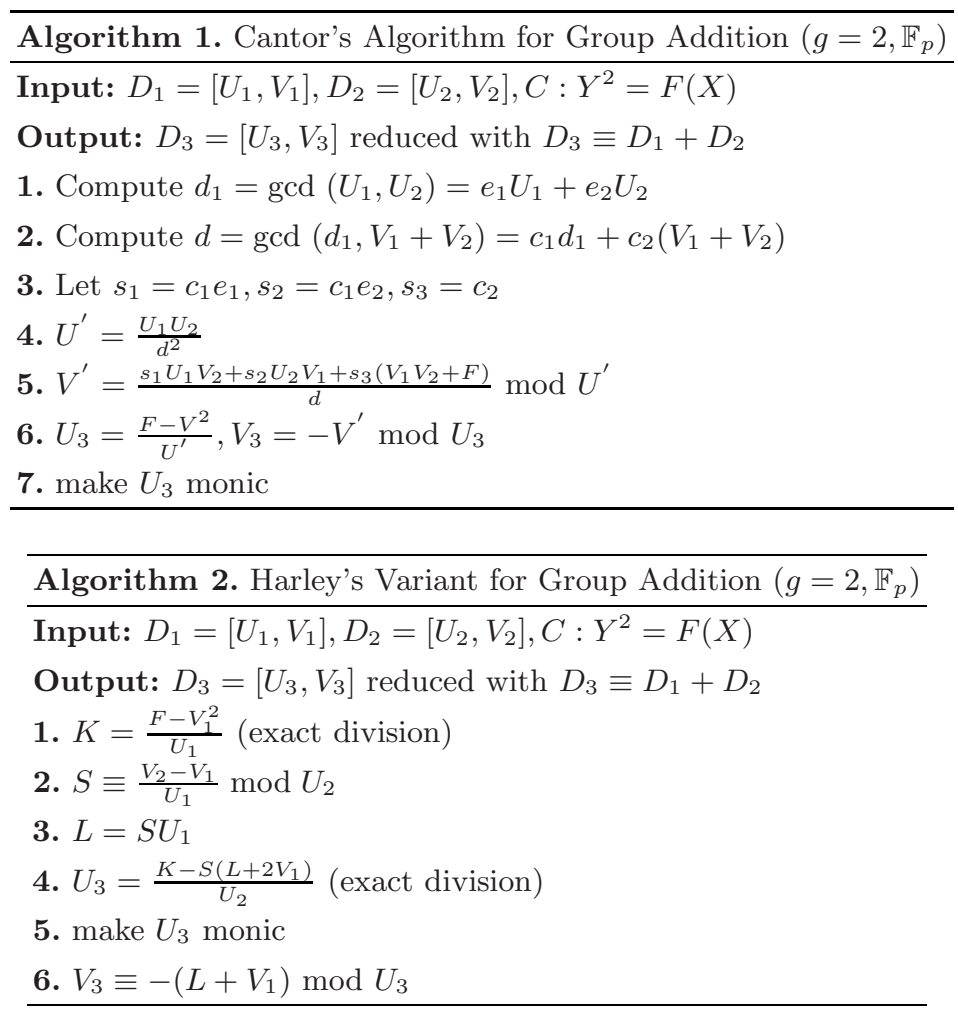

a group doubling. To derive explicit formulae, we first study all the exceptional cases during the computation $2 D_{1}+D_{2}$ based on the properties of the input divisor classes and the immediate result $D^{\prime}$. We then determine how many field operations are required to calculate $2 D_{1}+D_{2}$ in one step in the most frequent case. Furthermore, we also propose a variant of our basic algorithm by using Montgomery's trick to compute the two inversions simultaneously at cost of some multiplications, which will be more efficient whenever a field inversion is more expensive than about sixteen field multiplications.

\subsection{Explicit Formulae in Exceptional Cases}

In this subsection we discuss all the exceptional cases appearing in the procedure of calculating $2 D_{1}+D_{2}$. Suppose that $D_{1}=\left[U_{1}, V_{1}\right]$ and $D_{2}=\left[U_{2}, V_{2}\right]$ are two reduced divisor classes as the inputs of the composition step of the Cantor's algorithm. The final output is $D_{3}=2 D_{1}+D_{2}=\left[U_{3}, V_{3}\right]$. We need to distinguish the following cases:

1. $U_{1}$ is of degree zero, this is only possible in the case $\left[U_{1}, V_{1}\right]=[1,0]$, i.e. $D_{1}$ is the zero element of the divisor class group. The result of $2 D_{1}+D_{2}$ is the second class $D_{2}=\left[U_{2}, V_{2}\right]$. 
2. $U_{1}$ is of degree one and $U_{2}$ has degree zero, one or full degree. Let $U_{1}=X+u_{10}$ and $V_{1}=v_{10} \neq 0$ is a constant.

A. Assume $\operatorname{deg} U_{2}=0$, i.e., $D_{2}$ is the zero element of the divisor class group. Therefore, the result of $2 D_{1}+D_{2}$ is $2 D_{1}$ and we double the divisor $D_{1}$ with $1 I+4 M+1 S$ to obtain

$$
\begin{aligned}
& U_{3}=U_{1}^{2}=\left(X+u_{10}\right)^{2}, \\
& V_{3}=\frac{F^{\prime}\left(-u_{10}\right)\left(X+u_{10}\right)}{2 v_{10}}+v_{10} .
\end{aligned}
$$

B. Assume $\operatorname{deg} U_{2}=1$, i.e., $U_{2}=X+u_{20}$ and $V_{2}=v_{20} \neq 0$ is a constant.

i. If $U_{1}=U_{2}$ and $V_{1}=-V_{2}$, the result of $D_{1}+D_{2}$ is the zero element $[1,0]$. Hence, we get $2 D_{1}+D_{2}=[1,0]+D_{1}=D_{1}$;

ii. If $U_{1}=U_{2}$ and $V_{1}=V_{2}$, the result of $2 D_{1}+D_{2}$ is $3 D_{1}$, which can be computed with $1 I+12 M+4 S$ (See Table 5 in the appendix).

iii. Otherwise the result of $D_{1}+D_{2}$ is $\left[U^{\prime}, V^{\prime}\right]$ where

$$
\begin{aligned}
U^{\prime} & =U_{1} U_{2}=\left(X+u_{10}\right)\left(X+u_{20}\right), \\
V^{\prime} & =\frac{\left(v_{20}-v_{10}\right) X+v_{20} u_{10}-v_{10} u_{20}}{u_{10}-u_{20}} .
\end{aligned}
$$

And then we use Table 6 (see the appendix) to obtain $2 D_{1}+D_{2}$ in an additional $1 I+18 M+4 S$.

C. Assume deg $U_{2}=2$, i.e. $U_{2}=X^{2}+u_{21} X+u_{20}$ and $V_{2}=v_{21} X+v_{20}$. Then the corresponding divisors are given by $D_{1}=\left(P_{1}\right)-\left(P_{\infty}\right)$ and $D_{2}=\left(P_{2}\right)+\left(P_{3}\right)-2\left(P_{\infty}\right)$, with $P_{i} \neq P_{\infty}(i=1,2,3)$.

i. If $U_{2}\left(-u_{10}\right) \neq 0$ then $P_{1}$ and $-P_{1}$ do not occur in $D_{2}$. This case is dealt with Table 7 (see the appendix). We can obtain $2 D_{1}+D_{2}$ at the cost of $I+28 M+4 S$.

ii. Otherwise if $V_{2}\left(-u_{10}\right)=-v_{10}$ the $-P_{1}$ occurs in $D_{2}$ and the result of $D_{1}+D_{2}$ is $D^{\prime}=\left[U^{\prime}, V^{\prime}\right]=\left[X+u_{21}-u_{10}, v_{21}\left(-u_{21}+u_{10}\right)+v_{20}\right]$ because $-u_{21}$ equals the sum of the $x$-coordinates of the points. And then we compute $D_{3}$ using $(2)$, unless $D_{2}=2\left(-P_{1}\right)-2\left(P_{\infty}\right)$ where we can obtain $D_{3}=2 D_{1}+D_{2}=[1,0]$.

iii. The remaining case is that $P_{1}$ occurs in $D_{2}$. If $D_{2}=2\left(P_{1}\right)-2\left(P_{\infty}\right)=$ $2 D_{1}$, which holds if $u_{21}=2 u_{10}$ and $u_{20}=u_{10}^{2}$, then we have $2 D_{1}+$ $D_{2}=2 D_{2}$. Therefore, we obtain $D_{3}$ by doubling a class $D_{2}$ of order different from 2 and with first polynomial of full degree as in 3.A. Otherwise we first use Table 6 (see the appendix) to compute $D^{\prime}=$ $\left[U^{\prime}, V^{\prime}\right]=\left[X+u_{1}^{\prime} X+u_{0}^{\prime}, v_{1}^{\prime} X+v_{0}^{\prime}\right]=D_{1}+D_{2}$ with $1 I+18 M+4 S$ and then differentiate the following three cases to obtain $D_{3}=D^{\prime}+D_{1}$ : a. If $U^{\prime}\left(-u_{10}\right) \neq 0$ then $P_{1}$ and $-P_{1}$ do not occur in the support set of $D^{\prime}$. In this case, $D_{3}$ can be calculated with the explicit addition formula of the case of $\operatorname{deg} U_{1}=1$ and $\operatorname{deg} U_{2}=2$ in [16] at the cost of $1 I+10 M+S$. 
b. Otherwise If $V^{\prime}\left(-u_{10}\right)=-v_{10}$ then the $-P_{1}$ occurs in the support set of $D^{\prime}$. In this case, $D_{3}=\left[X+u_{1}^{\prime}-u_{10}, v_{1}^{\prime}\left(-u_{1}^{\prime}+u_{10}\right)+v_{0}^{\prime}\right]$.

c. The remaining case is that $P_{1}$ occurs in $D^{\prime}$. This case can be handled with steps $2 \sim 7$ of Table 6 (see the appendix) at the cost of $1 I+11 M+4 S$.

3. $U_{1}$ is of degree two and $U_{2}$ has degree zero, one or two. Let $U_{1}=X^{2}+$ $u_{11} X+u_{10}$ and $V_{1}=v_{11} X+v_{10}$. The corresponding divisor is given by $D_{1}=\left(P_{1}\right)+\left(P_{2}\right)-2\left(P_{\infty}\right)$ with $P_{i} \neq P_{\infty}(\mathrm{i}=1,2)$.

A. Assume $\operatorname{deg} U_{2}=0$, i.e. $D_{2}$ is the zero element of the divisor class group. Therefore, the result of $2 D_{1}+D_{2}$ is $2 D_{1}$ and we are in the case of doubling a divisor of order different from 2 and with first polynomial of full degree. Again we need to consider two subcases depending on wether a point $P_{i}$ in the support has order 2 . The point $P_{i}=\left(x_{i}, y_{i}\right)$ is equal to its opposite if and only if $y_{i}=0$. To check for this case we compute the resultant of $U_{1}$ and $V_{1}$.

i. If $\operatorname{res}\left(U_{1}, V_{1}\right) \neq 0$ then we are in the usual case where both points are not equal to their opposite. This can be computed with the doubling explicit formula of the most frequent case in [16].

ii. Otherwise we compute the $\operatorname{gcd}\left(U_{1}, V_{1}\right)=\left(X-x_{i}\right)$ to get the coordinate of $P_{i}$ and double the divisor $\left[X+u_{11}+x_{i}, v_{11}\left(-u_{11}-x_{i}\right)+v_{10}\right]$ to obtain $2 D_{1}=2\left(P_{j}\right)-2\left(P_{\infty}\right)(j \neq i)$ with $(1)$.

$\mathbb{B}$. Assume $\operatorname{deg} U_{2}=1$, i.e. $U_{2}=X+u_{20}$ and $V_{2}=v_{20} \neq 0$ is a constant. The corresponding divisor is given by $D_{2}=\left(P_{3}\right)-\left(P_{\infty}\right)$ with $P_{3} \neq P_{\infty}$.

i. If $U_{1}\left(-u_{20}\right) \neq 0$ then $P_{3}$ and $-P_{3}$ do not occur in $D_{1}$. This case is dealt with Table 8 (see the appendix). We can obtain $2 D_{1}+D_{2}$ at the cost of $I+46 M+7 S$.

ii. Otherwise if $V_{1}\left(-u_{20}\right)=-v_{10}$ then $-P_{3}$ occurs in $D_{1}$ and the result of $D_{1}+D_{2}$ is $D^{\prime}=\left[U^{\prime}, V^{\prime}\right]=\left[X+u_{11}-u_{20}, v_{11}\left(-u_{11}+u_{20}\right)+v_{10}\right]$ because $-u_{11}$ equals the sum of the $x$-coordinates of the points. And then we compute $D_{3}=D_{1}+D^{\prime}$ using steps $2 \sim 7$ of Table 6 (see the appendix) in an additional $1 I+11 M+4 S$.

iii. The remaining case is that $P_{3}$ occurs in $D_{1}$. If $D_{1}=2 D_{2}=2\left(P_{3}\right)-$ $2\left(P_{\infty}\right)$, which holds if $u_{11}=2 u_{20}$ and $u_{10}=u_{20}^{2}$, then we first use Table 5 (see the appendix) to compute $D^{\prime}=3 D_{2}$ with $1 I+12 M+4 S$. Otherwise we first obtain $D^{\prime}=D_{1}+D_{2}$ using Table [6 (see the appendix) with $1 I+18 M+4 S$. And then we consider the following two cases:

a. If $\operatorname{res}\left(U_{1}, U^{\prime}\right) \neq 0$ then there is no point in the support of $D_{1}$ which is equal to a point or its opposite in the support of $D^{\prime}$. We deal with this case with the addition explicit formula of the most frequent case in 16 .

b. If the above resultant is zero, then $D^{\prime}=\left(P_{1}\right)+\left(P_{3}\right)-2\left(P_{\infty}\right)$ or $D^{\prime}=\left(-P_{1}\right)+\left(P_{3}\right)-2\left(P_{\infty}\right)$. We first obtain $\operatorname{gcd}\left(U_{1}, U^{\prime}\right)=$ $\left(X-u_{p 1}\right)$. And then we calculate $D_{3}=D_{1}+D^{\prime}$ at cost of $1 I+32 M+3 S$ and $1 I+7 M$ with Table 9 (see the appendix) for these two subcases, respectively. 
C. Assume $\operatorname{deg} U_{2}=2$, i.e. $U_{2}=X^{2}+u_{21} X+u_{20}$ and $V_{2}=v_{21} X+v_{20}$. The corresponding divisor is given by $D_{2}=\left(P_{3}\right)+\left(P_{4}\right)-2\left(P_{\infty}\right)$ with $P_{i} \neq P_{\infty}(i=3,4)$.

i. Let $U_{1}=U_{2}$. This means that the $x$-coordinates of $P_{i}$ and $P_{i+2}$ $(i=1,2)$ are equal for an appropriate ordering.

a. If $V_{1} \equiv-V_{2} \bmod U_{1}$ then we obtain $2 D_{1}+D_{2}=D_{1}+[1,0]=D_{1}$.

b. If $V_{1}=V_{2}$ then we have $2 D_{1}+D_{2}=3 D_{1}$. We first double $D_{1}$ to get $D^{\prime}$ based on the two cases in 3.A. If the degree of $U^{\prime}$ is one, then we need to consider three subcases in 2.C.iii. Otherwise, we differentiate two subcases in 3. B.iii to compute $D_{3}$.

c. The remaining case is that $P_{i}=P_{i+2}$ and $P_{j} \neq P_{j+2}(i, j \in\{1,2\}$ and $i \neq j$ ) is the opposite of $P_{j+2}$. Without loss of generality, we assume $P_{1}=P_{3}$ and $P_{2} \neq P_{4}$ is the opposite of $P_{4}$. We first calculate $D^{\prime}=D_{1}+D_{2}=2\left(P_{1}\right)-2\left(P_{\infty}\right)$ by using (1) to double the divisor class $\left[X-\left(v_{10}-v_{20}\right) /\left(v_{21}-v_{11}\right), V_{1}\left(\left(v_{10}-v_{20}\right) /\left(v_{21}-\right.\right.\right.$ $\left.\left.\left.v_{11}\right)\right)\right]$. And then we calculate $D_{3}=D^{\prime}+D_{1}$ by considering two subcases in 3.B.iii.

ii. For the remainder cases $U_{1} \neq U_{2}$, the following possibilities may appear:

a. If $\operatorname{res}\left(U_{1}, U_{2}\right) \neq 0$ then there is no point in the support of $D_{1}$ which is equal to a point or its opposite in the support of $D_{2}$. We first only compute the first part $U^{\prime}$ of $D^{\prime}$ with the addition explicit formula of the most frequent case in [16. And then we require to consider the following three subcases:

$\mathbb{1}$. If the degree of $U^{\prime}$ is one, which appears when $s_{1}^{\prime}=0$ (see Table 11), we first calculate the second part $V^{\prime}$ of $D^{\prime}$ with the addition explicit formula of the special case in [16. And then we need to consider three subcases in 2. C.iii to compute $D_{3}$.

2. If $\operatorname{deg} U^{\prime}=2$ and $\operatorname{res}\left(U_{1}, U^{\prime}\right)=0$, we first calculate the second part $V^{\prime}$ of $D^{\prime}$ with the addition explicit formula of the most frequent case in [16]. And then we compute $D_{3}$ with Table 9 (see the appendix).

3. The remainder case is $\operatorname{deg} U^{\prime}=2$ and $\operatorname{res}\left(U_{1}, U^{\prime}\right) \neq 0$. This is the most frequent case and we will deal with this case in the next subsection.

b. If $\operatorname{res}\left(U_{1}, U_{2}\right)=0$ then we first compute $D^{\prime}$ with Table 9 (see the appendix). If the degree of $U^{\prime}$ is one, then we need to consider three subcases in 2.C.iii. Otherwise, we differentiate two subcases in 3.B. iii to compute $D_{3}$.

Although there are many exceptional cases during the computation of $2 D_{1}+$ $D_{2}$, most frequently we are in the case of $\operatorname{gcd}\left(U_{1}, U_{2}\right)=\operatorname{gcd}\left(U_{1}, U^{\prime}\right)=1$ and $U^{\prime}$ being quadratic. Therefore, if we can reduce the computational complexity of explicit formulae in the most frequent case, the performance of the whole cyptosystem will be improved on average. 


\subsection{Explicit Formulae in the Most Frequent Case}

In this subsection, we present efficient explicit formulae for computing $2 D_{1}+D_{2}$ in the most frequent case where $U_{1}, U_{2}$ and $U^{\prime}$ are quadratic and gcd $\left(U_{1}, U_{2}\right)=$ $\operatorname{gcd}\left(U_{1}, U^{\prime}\right)=1$. Studying Harley's variant carefully, we note that the polynomial $V^{\prime}$ in the intermediate result $D^{\prime}$ only is used to obtain $S$ in the second group addition (see Step 2 in Algorithm 2). Therefore, when we substitute the expression of $V^{\prime}$ into $S$, we find the following important lemma which results in a significant speedup for calculating $2 D_{1}+D_{2}$.

Lemma 1. Let $C$ be a genus 2 HEC over $\mathbb{F}_{q}$ given by the equation (1). Assume that $D_{1}=\left[U_{1}, V_{1}\right], D_{2}=\left[U_{2}, V_{2}\right]$ and $D^{\prime}=\left[U^{\prime}, V^{\prime}\right]=D_{1}+D_{2}$ are reduced divisor classes in the Jacobian $\mathcal{J}_{C}\left(\mathbb{F}_{q}\right)$ of $C$ and satisfy that $U_{1}, U_{2}$ and $U^{\prime}$ are quadratic, and $\operatorname{gcd}\left(U_{1}, U_{2}\right)=\operatorname{gcd}\left(U_{1}, U^{\prime}\right)=1$. Let $S$ and $S^{\prime}$ satisfy the congruent relations: $S \equiv \frac{V_{2}-V_{1}}{U_{1}} \bmod U_{2}$ and $S^{\prime} \equiv \frac{V^{\prime}-V_{1}}{U_{1}} \bmod U^{\prime}$, then we have

$$
S^{\prime} \equiv-S-\frac{2 V_{1}}{U_{1}} \bmod U^{\prime}
$$

Proof. From Harley's variant (see Algorithm 2), we know that

$$
V^{\prime} \equiv-\left(S U_{1}+V_{1}\right) \bmod U^{\prime}
$$

Substitute $V^{\prime}$ into $S^{\prime}$, we obtain

$$
S^{\prime} \equiv \frac{V^{\prime}-V_{1}}{U_{1}} \equiv \frac{-S U_{1}-2 V_{1}}{U_{1}} \equiv-S-\frac{2 V_{1}}{U_{1}} \bmod U^{\prime} .
$$

Lemma 1 suggests that we can eliminate the computation of $V^{\prime}$ during the procedure of calculating $2 D_{1}+D_{2}$. Table 1 presents our new explicit formula (Basic Algorithm) for computing $2 D_{1}+D_{2}$ on a genus 2 HEC over $\mathbb{F}_{p}$ in the most frequent case.

Table 1. Explicit Formula for $2 D_{1}+D_{2}$ on a HEC of Genus 2 over $\mathbb{F}_{p}-$ Basic Version

\begin{tabular}{|c|l|c|}
\hline Input & Genus 2 HEC $C: Y^{2}=F(X), F=X^{5}+f_{3} X^{3}+f_{2} X^{2}+f_{1} X+f_{0} ;$ & \\
& Reduced Divisors $D_{1}=\left(U_{1}, V_{1}\right)$ and $D_{2}=\left(U_{2}, V_{2}\right)$, & \\
& $U_{1}=X^{2}+u_{11} X+u_{10}, V_{1}=v_{11} X+v_{10} ;$ & \\
& $U_{2}=X^{2}+u_{21} X+u_{20}, V_{2}=v_{21} X+v_{20} ;$ & \\
\hline Output & Reduced Divisor $D_{3}=\left(U_{3}, V_{3}\right)=2 D_{1}+D_{2} ;$ & Cost \\
\hline \hline Step & $U_{3}=X^{2}+u_{31} X+u_{30}, V_{3}=v_{31} X+v_{30} ;$ & $3 M, 1 S$ \\
\hline 1 & Expression & Compute the resultant $\boldsymbol{r}$ of $\boldsymbol{U}_{\mathbf{1}}$ and $\boldsymbol{U}_{\mathbf{2}}:$ \\
\hline 2 & $i_{1}=u_{21}-u_{11}, w=u_{10}-u_{20}, i_{0}=i_{1} u_{21}+w, r=i_{0} w+i_{1}^{2} u_{20} ;$ & \\
\hline & Compute the pseudo-inverse $\boldsymbol{I}=\boldsymbol{i}_{\mathbf{1}} \boldsymbol{X}+\boldsymbol{i}_{\mathbf{0}} \equiv \boldsymbol{r} / \boldsymbol{U}_{\mathbf{1}} \mathbf{m o d} \boldsymbol{U}_{\mathbf{2}}:$ & - \\
\hline
\end{tabular}


Table 1. (continued)

\begin{tabular}{|c|c|c|}
\hline 3 & $\begin{array}{l}\text { Compute } \boldsymbol{S}^{\prime}=\boldsymbol{s}_{\mathbf{1}}^{\prime} \boldsymbol{X}+\boldsymbol{s}_{\mathbf{0}}^{\prime}=\boldsymbol{r} \boldsymbol{S} \equiv\left(\boldsymbol{V}_{\mathbf{2}}-\boldsymbol{V}_{\mathbf{1}}\right) \boldsymbol{I} \bmod \boldsymbol{U}_{\mathbf{2}}: \\
w_{0}=v_{20}-v_{10}, w_{1}=v_{21}-v_{11}, w_{2}=i_{0} w_{0}, w_{3}=i_{1} w_{1}, s_{0}^{\prime}=w_{2}-u_{20} w_{3} ; \\
s_{1}^{\prime}=\left(i_{0}+i_{1}\right)\left(w_{0}+w_{1}\right)-w_{2}-w_{3}\left(1+u_{21}\right) ; \text { If } s_{1}^{\prime}=0, \text { see 3.C.ii.a. } \mathbb{1} .\end{array}$ & $5 M$ \\
\hline 4 & $\begin{array}{l}\text { Compute } \boldsymbol{S}^{\prime \prime}=\boldsymbol{X}+\boldsymbol{s}_{\mathbf{0}} / \boldsymbol{s}_{\mathbf{1}}=\boldsymbol{X}+\boldsymbol{s}_{\mathbf{0}}^{\prime} / \boldsymbol{s}_{1}^{\prime} \text { and } \boldsymbol{s}_{\mathbf{1}}: \\
w_{1}=\left(r s_{1}^{\prime}\right)^{-1}\left(=1 / r^{2} s_{1}\right), w_{2}=r w_{1}\left(=1 / s_{1}^{\prime}\right), w_{3}=r w_{2}\left(=1 / s_{1}\right) \\
w_{4}=w_{3}^{2}, w_{5}=s_{1}^{\prime} w_{1}, s_{0}^{\prime \prime}=s_{0}^{\prime} w_{2}\end{array}$ & $1 I, 5 M, 1 S$ \\
\hline 5 & $\begin{array}{l}\text { Compute } \boldsymbol{U}^{\prime}=\left(\boldsymbol{s}\left(\boldsymbol{l}+\mathbf{2} \boldsymbol{V}_{\mathbf{1}}\right)-\boldsymbol{k}\right) / \boldsymbol{U}_{\mathbf{2}}=\boldsymbol{X}^{\mathbf{2}}+\boldsymbol{u}_{\mathbf{1}}^{\prime} \boldsymbol{X}+\boldsymbol{u}_{\mathbf{0}}^{\prime}: \\
\frac{u_{0}^{\prime}=\left(s_{0}^{\prime \prime}-u_{21}\right)\left(s_{0}^{\prime \prime}-i_{1}\right)+u_{11} s_{0}^{\prime \prime}+w+2 v_{11} w_{3}+\left(u_{11}+u_{21}\right) w_{4}}{u_{1}^{\prime}=2 s_{0}^{\prime \prime}-i_{1}-w_{4}}\end{array}$ & $4 M$ \\
\hline 6 & $\begin{array}{l}\text { Compute the resultant } \tilde{\boldsymbol{r}} \text { of } \boldsymbol{U}_{\mathbf{1}} \text { and } \boldsymbol{U}^{\prime}: \\
\tilde{i}_{1}=u_{1}^{\prime}-u_{11}, \tilde{w}=u_{10}-u_{0}^{\prime}, \tilde{i}_{0}=\tilde{i}_{1} u_{1}^{\prime}+\tilde{w}, \tilde{r}\end{array}=\tilde{i}_{0} \tilde{w}+\tilde{i}_{1}^{2} u_{0}^{\prime}$ & $4 M, 1 S$ \\
\hline 7 & Compute the pseudo-inverse $\tilde{I}=\tilde{i}_{1} X+\tilde{i}_{0} \equiv \tilde{r} / U_{1} \bmod U^{\prime}:$ & - \\
\hline 8 & $\begin{array}{l}\text { Compute } \tilde{\boldsymbol{S}}^{\prime}=\tilde{\boldsymbol{s}}_{1}^{\prime} \boldsymbol{X}+\tilde{\boldsymbol{s}}_{\mathbf{0}}^{\prime}=\tilde{\boldsymbol{r}} \tilde{\boldsymbol{S}} \equiv-\tilde{\boldsymbol{r}} \boldsymbol{S}^{\prime} / \boldsymbol{r}-\mathbf{2} \boldsymbol{V}_{\mathbf{1}} \tilde{\boldsymbol{I}} \bmod \boldsymbol{U}^{\prime}: \\
\tilde{r}^{\prime}=\tilde{r} w_{5}, \tilde{w}_{0}=\tilde{i}_{0} v_{10}, \tilde{w}_{1}=\tilde{i}_{1} v_{11}, \tilde{s}_{0}^{\prime}=-\left[\tilde{r}^{\prime} s_{0}^{\prime}+2\left(\tilde{w}_{0}-u_{0}^{\prime} \tilde{w}_{1}\right)\right] ; \\
\tilde{s}_{1}^{\prime}=-\left[\tilde{r}^{\prime} s_{1}^{\prime}+2\left(\left(\tilde{i}_{0}+\tilde{i}_{1}\right)\left(v_{10}+v_{11}\right)-\tilde{w}_{0}-\tilde{w}_{1}\left(1+u_{1}^{\prime}\right)\right)\right] ; \text { If } \tilde{s}_{1}^{\prime}=0, \text { see below }\end{array}$ & $7 M$ \\
\hline 9 & $\begin{array}{l}\text { Compute } \tilde{\boldsymbol{s}}^{\prime \prime}=\boldsymbol{X}+\tilde{s}_{0} / \tilde{s}_{1}=\boldsymbol{X}+\tilde{s}_{0}^{\prime} / \tilde{s}_{1}^{\prime} \text { and } \tilde{s}_{1}: \\
\tilde{w}_{1}=\left(\tilde{r} \tilde{s}_{1}^{\prime}\right)^{-1}\left(=1 / \tilde{r}^{2} \tilde{s}_{1}\right), \tilde{w}_{2}=\tilde{r} \tilde{w}_{1}\left(=1 / \tilde{s}_{1}^{\prime}\right), \tilde{w}_{3}=\tilde{s}_{1}^{\prime}{ }_{w} \tilde{w}_{1}\left(=\tilde{s}_{1}\right) \\
\tilde{w}_{4}=\tilde{r} \tilde{w}_{2}\left(=1 / \tilde{s}_{1}\right), \tilde{w}_{5}=\tilde{w}_{4}^{2}, \tilde{s}_{0}^{\prime \prime}=\tilde{s}_{0}^{\prime} \tilde{w}_{2}\end{array}$ & $1 I, 5 M, 2 S$ \\
\hline 10 & $\begin{array}{l}\text { Compute } \tilde{\boldsymbol{l}}^{\prime}=\tilde{\boldsymbol{S}}^{\prime \prime} \boldsymbol{u}_{\mathbf{1}}=\boldsymbol{X}^{\mathbf{3}}+\tilde{\boldsymbol{l}}_{\mathbf{2}}^{\prime} \boldsymbol{X}^{\mathbf{2}}+\tilde{l}_{1}^{\prime} \boldsymbol{X}+\tilde{\boldsymbol{l}}_{\mathbf{0}}^{\prime}: \\
\tilde{l}_{2}^{\prime}=u_{11}+\tilde{s}_{0}^{\prime \prime}, \tilde{l}_{1}^{\prime}=u_{11} \tilde{s}_{0}^{\prime \prime}+u_{10}, \tilde{l}_{0}^{\prime}=u_{10} \tilde{s}_{0}^{\prime \prime}\end{array}$ & $2 M$ \\
\hline 11 & $\begin{array}{l}\text { Compute } \boldsymbol{U}_{3}=\left(\tilde{\boldsymbol{s}}\left(\tilde{\boldsymbol{l}}^{\prime}+\mathbf{2} \boldsymbol{V}_{\mathbf{1}}\right)-\boldsymbol{k}\right) / \boldsymbol{U}^{\prime}=\boldsymbol{X}^{\mathbf{2}}+\boldsymbol{u}_{31} \boldsymbol{X}+\boldsymbol{u}_{\mathbf{3 0}}: \\
u_{30}=\left(\tilde{s}_{0}^{\prime \prime}-u_{1}^{\prime}\right)\left(\tilde{s}_{0}^{\prime \prime}-\tilde{i}_{1}\right)-u_{0}^{\prime}+\tilde{l}_{1}^{\prime}+2 v_{11} \tilde{w}_{4}+\left(u_{1}^{\prime}+u_{11}\right) \tilde{w}_{5} \\
u_{31}=2 \tilde{s}_{0}^{\prime \prime}-\tilde{i}_{1}-\tilde{w}_{5}\end{array}$ & $3 M$ \\
\hline 12 & $\begin{array}{l}\text { Compute } \boldsymbol{V}_{\mathbf{3}}=-\left(\tilde{\boldsymbol{l}}^{\prime}+\boldsymbol{V}_{\mathbf{1}}\right) \bmod \boldsymbol{U}_{\mathbf{3}}=\boldsymbol{v}_{\mathbf{3 1}} \boldsymbol{X}+\boldsymbol{v}_{\mathbf{3 0}}: \\
w_{1}=\tilde{l}_{2}^{\prime}-u_{31}, w_{2}=u_{31} w_{1}+u_{30}-\tilde{l}_{1}^{\prime}, v_{31}=w_{2} \tilde{w}_{3}-v_{11} \\
w_{2}=u_{30} w_{1}-\tilde{l}_{0}^{\prime}, v_{30}=w_{2} \tilde{w}_{3}-v_{10}\end{array}$ & $4 M$ \\
\hline Sum & $\tilde{s}_{1}^{\prime} \neq 0$ & $2 I, 42 M, 5 S$ \\
\hline 9 ' & $\begin{array}{l}\text { Compute } \tilde{s}_{0}: \\
\tilde{w}_{1}=\tilde{r}^{-1}, \tilde{s}_{0}=\tilde{s}_{0}^{\prime} \tilde{w}_{1}\end{array}$ & $1 I, 1 M$ \\
\hline $10^{\prime}$ & $\begin{array}{l}\text { Compute } U_{3}=\left(k-\tilde{s}\left(\tilde{l}+2 V_{1}\right)\right) / U^{\prime}=X+u_{30}: \\
u_{30}=-\left(u_{1}^{\prime}+u_{11}+\tilde{s}_{0}^{2}\right)\end{array}$ & $1 S$ \\
\hline $11^{\prime}$ & $\begin{array}{l}\text { Compute } \boldsymbol{V}_{\mathbf{3}}=-\left(\tilde{\boldsymbol{l}}+\boldsymbol{V}_{\mathbf{1}}\right) \bmod \boldsymbol{U}_{\mathbf{3}}=\boldsymbol{v}_{\mathbf{3 0}}: \\
w_{1}=\tilde{s}_{0}\left(u_{1}^{\prime}+u_{30}\right)+v_{11}, w_{2}=\tilde{s}_{0}+v_{10}, v_{30}=u_{0}^{\prime} w_{1}-w_{2} ;\end{array}$ & $2 M$ \\
\hline Sum & $\tilde{s}_{1}^{\prime}=0$ & $2 I, 31 M, 4 S$ \\
\hline
\end{tabular}

Our explicit formula of the basic version requires $2 I+42 M+5 S$ to calculate $2 D_{1}+D_{2}$ for genus 2 HECs over $\mathbb{F}_{p}$. However, the naive method which separately computes the two divisor class additions will cost $2 I+44 M+6 S$ [16]. Therefore, our improvements can save $2 M+1 S$ each time the operation $2 D_{1}+D_{2}$ is performed.

We note that there exist two inversions in the above explicit formula of the basic version. Therefore, we propose a variant of the basic algorithm where we delay the inversion in Step 4 of Table 1 and combine it with the inversion in Step 6 of Table 1 using Montgomery's trick of simultaneous inversions [8]. Table 2 presents the explicit formula for this variant of the basic algorithm. 
Table 2. Explicit Formula for $2 D_{1}+D_{2}$ on a HEC of Genus 2 over $\mathbb{F}_{p}$ - Variant

\begin{tabular}{|c|c|c|}
\hline Input & $\begin{array}{l}\text { Genus } 2 \text { HEC } C: Y^{2}=F(X), F=X^{5}+f_{3} X^{3}+f_{2} X^{2}+f_{1} X+f_{0} \\
\text { Reduced Divisors } D_{1}=\left(U_{1}, V_{1}\right) \text { and } D_{2}=\left(U_{2}, V_{2}\right) \\
U_{1}=X^{2}+u_{11} X+u_{10}, V_{1}=v_{11} X+v_{10} \\
U_{2}=X^{2}+u_{21} X+u_{20}, V_{2}=v_{21} X+v_{20}\end{array}$ & \\
\hline Output & $\begin{array}{l}\text { Reduced Divisor } D_{3}=\left(U_{3}, V_{3}\right)=2 D_{1}+D_{2} \\
U_{3}=X^{2}+u_{31} X+u_{30}, V_{3}=v_{31} X+v_{30}\end{array}$ & \\
\hline Step & Expression & Cost \\
\hline 1 & $\begin{array}{l}\text { Compute the resultant } \boldsymbol{r} \text { of } \boldsymbol{U}_{\mathbf{1}} \text { and } \boldsymbol{U}_{\mathbf{2}} \text { : } \\
i_{1}=u_{21}-u_{11}, w=u_{10}-u_{20}, i_{0}=i_{1} u_{21}+w, r=i_{0} w+i_{1}^{2} u_{20}\end{array}$ & $3 M, 1 S$ \\
\hline 2 & Compute the pseudo-inverse $I=i_{1} X+i_{0} \equiv r / U_{1} \bmod U_{2}$ : & - \\
\hline 3 & $\begin{array}{l}\text { Compute } \boldsymbol{S}^{\prime}=\boldsymbol{s}_{\mathbf{1}}^{\prime} \boldsymbol{X}+\boldsymbol{s}_{\mathbf{0}}^{\prime}=\boldsymbol{r} \boldsymbol{S} \equiv\left(\boldsymbol{V}_{\mathbf{2}}-\boldsymbol{V}_{\mathbf{1}}\right) \boldsymbol{I} \bmod \boldsymbol{U}_{\mathbf{2}}: \\
w_{0}=v_{20}-v_{10}, w_{1}=v_{21}-v_{11}, w_{2}=i_{0} w_{0}, w_{3}=i_{1} w_{1}, \boldsymbol{s}_{0}^{\prime}=w_{2}-u_{20} w_{3} \\
s_{1}^{\prime}=\left(i_{0}+i_{1}\right)\left(w_{0}+w_{1}\right)-w_{2}-w_{3}\left(1+u_{21}\right) ; \text { If } s_{1}^{\prime}=0, \text { see 3.C.ii.a. } \mathbb{1} .\end{array}$ & $5 M$ \\
\hline 4 & Monic $S^{\prime \prime}=X+s_{0} / s_{1}=X+s_{0}^{\prime} / s_{1}^{\prime}:$ & - \\
\hline 5 & $\begin{array}{l}\text { Compute } \boldsymbol{U}^{\prime}=\left(\boldsymbol{s}\left(\boldsymbol{l}+\mathbf{2} \boldsymbol{V}_{\mathbf{1}}\right)-\boldsymbol{k}\right) / \boldsymbol{U}_{\mathbf{2}}=\boldsymbol{X}^{\mathbf{2}}+\boldsymbol{u}_{\mathbf{1}}^{\prime} \boldsymbol{X}+\boldsymbol{u}_{\mathbf{0}}^{\prime}: \\
s_{q}=s_{1}^{\prime}, w_{1}=i_{1} s_{1}^{\prime}, w_{2}=s_{0}^{\prime}-w_{1}, R=r^{2}, u_{1}^{\prime}=s_{1}^{\prime}\left(s_{0}^{\prime}+w_{2}\right)-R ; \\
u_{0}^{\prime}=s_{0}^{\prime}\left(w_{2}-w_{1}\right)+i_{0} s_{q}+2 r v_{11} s_{1}^{\prime}+R\left(u_{11}+u_{21}\right)\end{array}$ & $7 M, 2 S$ \\
\hline 6 & $\begin{array}{l}\text { Compute the resultant } \tilde{\boldsymbol{r}} \text { of } \boldsymbol{U}_{\mathbf{1}} \text { and } \boldsymbol{U}^{\prime}: \\
\tilde{i}_{1}=u_{1}^{\prime}-u_{11} s_{q}, \tilde{w}=u_{10} s_{q}-u_{0}^{\prime}, \tilde{i}_{0}=u_{1}^{\prime} \tilde{i}_{1}+\tilde{w} s_{q}, \tilde{r}=\tilde{i}_{0} \tilde{w}+\tilde{i}_{1}^{2} u_{0}^{\prime} ;\end{array}$ & $6 M, 1 S$ \\
\hline 7 & Compute the pseudo-inverse $\tilde{I}=\tilde{i}_{1} X+\tilde{i}_{0} \equiv \tilde{r} / U_{1} \bmod U^{\prime}:$ & - \\
\hline 8 & $\begin{array}{l}\text { Compute } \tilde{\boldsymbol{S}}^{\prime}=\tilde{\boldsymbol{s}}_{1}^{\prime} \boldsymbol{X}+\tilde{\boldsymbol{s}}_{\mathbf{0}}^{\prime}=\tilde{\boldsymbol{r}} \tilde{\boldsymbol{S}} \equiv-\tilde{\boldsymbol{r}} \boldsymbol{S}^{\prime} / \boldsymbol{r}-\mathbf{2} \boldsymbol{V}_{\mathbf{1}} \tilde{\boldsymbol{I}} \bmod \boldsymbol{U}^{\prime}: \\
\tilde{w}_{0}=\tilde{i}_{0} v_{10}, \tilde{w}_{1}=\tilde{i}_{1} v_{11}, \tilde{w}_{2}=r s_{q}, \tilde{s}_{0}^{\prime}=-\left[\tilde{r} s_{0}^{\prime}+2 \tilde{w}_{2}\left(\tilde{w}_{0}-u_{0}^{\prime} \tilde{w}_{1}\right)\right] \\
\tilde{s}_{1}^{\prime}=-\left[\tilde{r} s_{1}^{\prime}+2 \tilde{w}_{2}\left(\left(\tilde{i}_{0}+\tilde{i}_{1} s_{q}\right)\left(v_{10}+v_{11}\right)-\tilde{w}_{0}-\tilde{w}_{1}\left(s_{q}+u_{1}^{\prime}\right)\right)\right] \\
\text { If } \tilde{s}_{1}^{\prime}=0, \text { see below }\end{array}$ & $11 M$ \\
\hline 9 & $\begin{array}{l}\text { Compute } \tilde{\boldsymbol{S}}^{\prime \prime}=\boldsymbol{X}+\tilde{\boldsymbol{s}}_{\mathbf{0}} / \tilde{\boldsymbol{s}}_{\mathbf{1}}=\boldsymbol{X}+\tilde{\boldsymbol{s}}_{\mathbf{0}}^{\prime} / \tilde{\boldsymbol{s}}_{\mathbf{1}}^{\prime} \text { and } \tilde{\boldsymbol{s}}_{\mathbf{1}}: \\
t_{1}=\tilde{r} \tilde{s}_{1}^{\prime}, t_{2}=\left(t_{1} \tilde{w}_{2}\right)^{-1}, t_{3}=\tilde{w}_{2} t_{2}, t_{4}=t_{1} t_{2}, t_{5}=r t_{4}, t_{6}=s_{q} t_{4} ; \\
\tilde{w}_{1}=r t_{3}, t_{7}=\left(t_{6} \tilde{s}_{1}^{\prime}\right)^{2}, \tilde{w}_{3}=t_{7} \tilde{w}_{1}, \tilde{w}_{4}=\tilde{r}^{2} \tilde{w}_{1}, \tilde{w}_{5}=\tilde{w}_{4}^{2}, \tilde{s}_{0}^{\prime \prime}=\tilde{r} \tilde{s}_{0}^{\prime} t_{3} ;\end{array}$ & $1 I, 12 M, 3 S$ \\
\hline 10 & 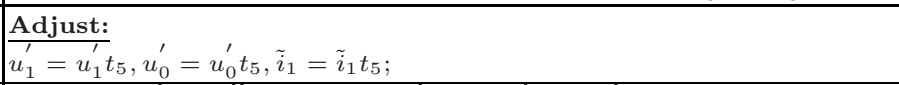 & $3 M$ \\
\hline 11 & $\begin{array}{l}\text { Compute } \tilde{l}^{\prime}=\tilde{\boldsymbol{S}}^{\prime \prime} \boldsymbol{u}_{\mathbf{1}}=\boldsymbol{X}^{\mathbf{3}}+\tilde{\boldsymbol{l}}_{\mathbf{2}}^{\prime} \boldsymbol{X}^{\mathbf{2}}+\tilde{\boldsymbol{l}}_{1}^{\prime} \boldsymbol{X}+\tilde{\boldsymbol{l}}_{\mathbf{0}}^{\prime}: \\
\tilde{l}_{2}^{\prime}=u_{11}+\tilde{s}_{0}^{\prime \prime}, \tilde{l}_{1}^{\prime}=u_{11} \tilde{s}_{0}^{\prime \prime}+u_{10}, \tilde{l}_{0}^{\prime}=u_{10} \tilde{s}_{0}^{\prime \prime}\end{array}$ & $2 M$ \\
\hline 12 & $\begin{array}{l}\text { Compute } \boldsymbol{U}_{\mathbf{3}}=\left(\tilde{\boldsymbol{s}}\left(\tilde{\boldsymbol{l}}^{\prime}+\mathbf{2} \boldsymbol{V}_{\mathbf{1}}\right)-\boldsymbol{k}\right) / \boldsymbol{U}^{\prime}=\boldsymbol{X}^{\mathbf{2}}+\boldsymbol{u}_{\mathbf{3 1}} \boldsymbol{X}+\boldsymbol{u}_{\mathbf{3 0}}: \\
u_{30}=\left(\tilde{s}_{0}^{\prime \prime}-u_{1}^{\prime}\right)\left(\tilde{s}_{0}^{\prime \prime}-\tilde{i}_{1}\right)-u_{0}^{\prime}+\tilde{l}_{1}^{\prime}+2 v_{11} \tilde{w}_{4}+\left(u_{1}^{\prime}+u_{11}\right) \tilde{w}_{5} ; \\
u_{31}=2 \tilde{s}_{0}^{\prime \prime}-\tilde{i}_{1}-\tilde{w}_{5} ;\end{array}$ & $3 M$ \\
\hline 13 & $\begin{array}{l}\text { Compute } \boldsymbol{V}_{\mathbf{3}}=-\left(\tilde{\boldsymbol{l}}^{\prime}+\boldsymbol{V}_{\mathbf{1}}\right) \bmod \boldsymbol{U}_{\mathbf{3}}=\boldsymbol{v}_{\mathbf{3 1}} \boldsymbol{X}+\boldsymbol{v}_{\mathbf{3 0}}: \\
w_{1}=\tilde{l}_{2}^{\prime}-u_{31}, w_{2}=u_{31} w_{1}+u_{30}-\tilde{l}_{1}^{\prime}, v_{31}=w_{2} \tilde{w}_{3}-v_{11} \\
w_{2}=u_{30} w_{1}-\tilde{l}_{0}^{\prime}, v_{30}=w_{2} \tilde{w}_{3}-v_{10}\end{array}$ & $4 M$ \\
\hline Sum & $\tilde{s}_{1}^{\prime} \neq 0$ & $I, 56 M, 7 S$ \\
\hline 9 ' & 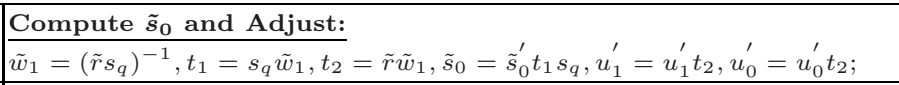 & $1 I, 7 M$ \\
\hline $10^{\prime}$ & $\begin{array}{l}\text { Compute } U_{3}=\left(\boldsymbol{k}-\tilde{s}\left(\tilde{l}+2 V_{1}\right)\right) / U^{\prime}=\boldsymbol{X}+\boldsymbol{u}_{30}: \\
u_{30}=-\left(u_{1}^{\prime}+u_{11}+\tilde{s}_{0}^{2}\right) ;\end{array}$ & $1 S$ \\
\hline $11^{\prime}$ & $\begin{array}{l}\text { Compute } \boldsymbol{V}_{\mathbf{3}}=-\left(\tilde{\boldsymbol{l}}+\boldsymbol{V}_{\mathbf{1}}\right) \bmod \boldsymbol{U}_{\mathbf{3}}=\boldsymbol{v}_{\mathbf{3 0}}: \\
w_{1}=\tilde{s}_{0}\left(u_{1}^{\prime}+u_{30}\right)+v_{11}, w_{2}=\tilde{s}_{0}+v_{10}, v_{30}=u_{0}^{\prime} w_{1}-w_{2} ;\end{array}$ & $2 M$ \\
\hline Sum & $\tilde{s}_{1}^{\prime}=0$ & $I, 38 M, 6 S$ \\
\hline
\end{tabular}


In Table 2, the variant of the basic algorithm needs $I+56 M+7 S$ to calculate $2 D_{1}+D_{2}$ for genus 2 HECs over $\mathbb{F}_{p}$. Compared to our explicit formula of the basic version, we trade $1 I$ for $14 M+2 S$. Therefore, when we implement genus 2 HECC on some application environments where a field inversion is more expensive than fourteen field multiplications and two field squarings, the variant in Table 2 will be faster than the basic algorithm in Table 1.

\subsection{Cost of the NAF Scalar Multiplication}

The above trick of efficiently computing $2 D_{1}+D_{2}$ has found important applications in some scalar multiplication algorithms such as NAF, JSF and so on 7. In this subsection, we only compare the average cost per bit scalar when implementing NAF scalar multiplication algorithm with the naive method and our newly derived formulae, respectively, because the NAF scalar multiplication algorithm will be used in our implementation in the next section. The results of comparisons are listed in the following Table 3 (The pre- and post-computations are neglected as in [7]).

Table 3. Average Cost Per Bit for NAF on Genus 2 HECs over $\mathbb{F}_{p}$

\begin{tabular}{|c|c|c|c|}
\hline Method & Cost of $2 D_{1}+D_{2}$ & Cost per bit scalar & $S=0.8 M$ \\
\hline \hline Naive & $2 I+44 M+6 S$ & $\frac{4}{3} I+\frac{88}{3} M+\frac{16}{3} S$ & $1.33 I+33.6 M$ \\
\hline Basic Algorithm (Table 1) & $2 I+42 M+5 S$ & $\frac{4}{3} I+\frac{86}{3} M+5 S$ & $1.33 I+32.67 M$ \\
\hline Variant (Table 2) & $1 I+56 M+7 S$ & $1 I+\frac{100}{3} M+\frac{17}{3} S$ & $1 I+37.87 M$ \\
\hline
\end{tabular}

From Table 3, we can see clearly that our basic algorithm saves about $2.8 \%$ cost for per bit scalar compared to the naive method and the break-even point of the performance between the basic algorithm and the variant is still when one inversion is equivalent to about sixteen field multiplications.

\section{Implementation Results}

We implement the proposed algorithms on a Pentium-4@2.8GHz processor and with $\mathrm{C}$ programming language in order to check the correctness and test the performance of our explicit formulae. Microsoft Developer Studio 6 is used for compilation and debugging. For genus $2 \mathrm{HECC}$ over $\mathbb{F}_{q}$, the most efficient attack is Pollard's Rho algorithm which takes $O\left(\sqrt{\# \mathcal{J}_{C}\left(\mathbb{F}_{q}\right)}\right)$ group operations. This means that for genus 2 HECC a 80 -bit finite field is enough to achieve the same security level as 160-bit ECC. Considering the security and efficiency of the implementation, we choose a Mersenne prime $p=2^{89}-1$ as the characteristic of the prime field $\mathbb{F}_{p}$ and develop a fast library for the required field and group operations. The implementation of $\mathbb{F}_{p}$-arithmetic is basically due to [59] and further optimized by using the idea in 12 to yield a fast modular reduction 
Table 4. Timings of Group Operation on Genus 2 HECs over $\mathbb{F}_{2^{89}-1}$

\begin{tabular}{|c|c|c|c|}
\hline Method & $\begin{array}{c}2 D_{1}+D_{2} \\
\text { in } \mu s\end{array}$ & $\begin{array}{c}\text { Scalar Multiplication } \\
\text { in } m s\end{array}$ & $\begin{array}{c}\text { Performance } \\
\text { Improvement }\end{array}$ \\
\hline \hline Naive & 23.1 & 2.85 & - \\
\hline Basic Algorithm (Table 1) & 21.7 & 2.78 & $2.46 \%$ \\
\hline Variant (Table 2) & 16.4 & 2.48 & $12.98 \%$ \\
\hline
\end{tabular}

procedure. Table 4 summarizes our implementation results and comparisons for the group operation $2 D_{1}+D_{2}$ and the NAF scalar multiplication algorithm.

The experimental results of Table 4 show that when compared to the implementation with the naive method the performance of genus 2 HECC can be improved by $2.46 \%$ and $12.98 \%$ with our basic algorithm and the variant, respectively. Furthermore, due to the high $M I$-ratio (the ratio of the timing of one inversion to one multiplication) in the target processor, the variant is about $10 \%$ faster than the basic algorithm.

\section{Conclusion}

In this paper, we propose the efficient algorithms for computing $2 D_{1}+D_{2}$ in one step for genus 2 HECs over prime fields. Our basic algorithm is the direct generalization of Eisenträger et al.s' idea, which can save $2 M+1 S$ compared with the naive method in the most frequent case. The performance of the variant will be better than that of the basic algorithm whenever a field inversion is more expensive than about sixteen field multiplications. Based our new explicit formulae, we analyze the average cost of per bit scalar in the NAF scalar multiplication algorithm and implement fast genus $2 \mathrm{HECC}$ over $\mathbb{F}_{2^{89}-1}$. The experimental results show that we can obtain up to $13 \%$ performance gain when implementing genus $2 \mathrm{HECC}$ with our newly derived explicit formulae.

\section{References}

1. Avanzi, R.M.: Aspects of Hyperelliptic Curves over Large Prime Fields in Software Implementations. In: Joye, M., Quisquater, J.-J. (eds.) CHES 2004. LNCS, vol. 3156, pp. 148-162. Springer, Heidelberg (2004)

2. Avanzi, R.M.: The Complexity of Certain Multi-Exponentiation Techniques in Cryptography. Journal of Cryptology 18(4), 357-373 (2005)

3. Avanzi, R.M., Cohen, H., Doche, C., Frey, G., Lange, T., Nguyen, K., Vercauteren, F.: Handbook of Elliptic and Hyperelliptic Curve Cryptography. Chapman \& Hall/CRC, Boca Raton, Florida, USA (2006)

4. Avanzi, R.M., Thériault, N., Wang, Z.: Rethinking Low Genus Hyperelliptic Jacobian Arithmetic over Binary Fields: Interplay of Field Arithmetic and Explicit Formulae, Centre for Applied Cryptographic Research (CACR) Technical Reports, CACR 2006-07, available at http://www.cacr.math.uwaterloo.ca/ 
5. Bailey, D.V., Paar, C.: Optimal Extension Fields for Fast Arithmetic in Public-Key Algorithms. In: Krawczyk, H. (ed.) CRYPTO 1998. LNCS, vol. 1462, pp. 472-485. Springer, Heidelberg (1998)

6. Cantor, D.: Computing in Jacobian of a Hyperelliptic Curve. Mathematics of Computation 48(177), 95-101 (1987)

7. Ciet, M., Joye, M., Lauter, K., Montgomery, L.: Trading Inversions for Multiplications in Elliptic Curve Cryptography. Design, Codes and Cryptography 39, 189-206 (2006)

8. Cohen, H.: A Course in Computational Algebraic Number Theory. Graduate Texts in Math., vol. 138. Springer, Berlin (1993) (fourth corrected printing, 2000)

9. Hankerson, D., Menezes, A., Vanstone, S.: Guide to Elliptic Curve Cryptography. Springer, New York (2004)

10. Eisenträger, K., Lauter, K., Montgomery, P.L.: Fast Elliptic Arithmetic and Improved Weil Pairing Evaluation. In: Joye, M. (ed.) CT-RSA 2003. LNCS, vol. 2612, pp. 343-354. Springer, Heidelberg (2003)

11. Gaudry, P., Harley, R.: Counting Points on Hyperelliptic Curves over Finite Fields. In: Bosma, W. (ed.) Algorithmic Number Theory. LNCS, vol. 1838, pp. 297-312. Springer, Heidelberg (2000)

12. Gonda, M., Matsuo, K., Aoki, K., Chao, J., Tsujii, S.: Improvements of Addition Algorithm on Genus 3 Hyperelliptic Curves and Their Implementation. IEICE Transactions on Fundamentals of Electronics, Communications and Computer Science E88-A(1), 89-96 (2005)

13. Gordon, D.M.: A Survey of Fast Exponentiation Methods. Journal of Algorithms 27(1), 129-146 (1998)

14. Koblitz, N.: A Family of Jacobian Suitable for Discrete Log Cryptosystems. In: Goldwasser, S. (ed.) CRYPTO 1988. LNCS, vol. 403, pp. 94-99. Springer, Heidelberg (1990)

15. Koblitz, N.: Hyperelliptic Cryptosystems. Journal of Cryptology 1(3), 129-150 (1989)

16. Lange, T.: Formulae for Arithmetic on Genus 2 Hyperelliptic Curves. Applicable Algebra in Engineering, Communication and Computing 15(5), 295-328 (2005)

17. Lange, T., Stevens, M.: Efficient Doubling for Genus Two Curves over Binary Fields. In: Handschuh, H., Hasan, M.A. (eds.) SAC 2004. LNCS, vol. 3357, pp. 170-181. Springer, Heidelberg (2004)

18. Menezes, A., Wu, Y., Zuccherato, R.: An Elementary Introduction to Hyperelliptic Curve, Centre for Applied Cryptographic Research (CACR) Technical Reports, CORR 1996-19, available at http://www.cacr.math.uwaterloo.ca/

19. Mumford, D.: Tata Lectures on Theta II. Prog. Math. 43, Birkhäuser (1984)

20. Nagao, K.: Improving Group Law Algorithms for Jacobians of Hyperelliptic Curves. In: Bosma, W. (ed.) Algorithmic Number Theory. LNCS, vol. 1838, pp. 439-448. Springer, Heidelberg (2000)

21. Wollinger, T.: Software and Harware Implementation of Hyperelliptic Curve Cryptosystems, PhD. thesis, Department of Electrical Engineering and Information Sciences, Ruhr-Universitäet Bochum, Bochum, Germany (2004)

22. Wollinger, T., Pelzl, J., Paar, C.: Cantor versus Harley: Optimization and Analysis of Explicit Formulae for Hyperelliptic Curve Cryptosystems. IEEE Transactions on Computers 54(7), 861-872 (2005) 


\section{Appendix: Explicit Formulae in Exceptional Cases}

In this appendix, we give the explicit addition formulae for the exceptional cases during the computation procedure of $2 D_{1}+D_{2}$, which have been discussed in detail in subection 3.1. These cases usually appear with a very low probability and therefore have not important influence on the performance of genus 2 HECC. Tables 5 to 9 list the detailed steps and the corresponding cost of the group addition in the exceptional cases. In Tables 5 to 9. $\mathrm{ADD}^{i+j \rightarrow k}$ denotes the divisor class addition $D_{3}=\left[U_{3}, V_{3}\right]=D_{1}+D_{2}=\left[U_{1}, V_{1}\right]+\left[U_{2}, V_{2}\right]$, and $\mathrm{TRI}^{i \rightarrow k}$ denotes the divisor class tripling $D_{3}=\left[U_{3}, V_{3}\right]=3 D_{1}=3\left[U_{1}, V_{1}\right]$, where $i, j$ and $k$ are the degrees of $U_{1}, U_{2}$ and $U_{3}$, respectively.

Table 5. Explicit Formula for $3 D_{1}$ on a HEC of Genus 2 over $\mathbb{F}_{p}$ : $\mathrm{TRI}^{1 \rightarrow 2}$

\begin{tabular}{|c|c|c|}
\hline Input & $\begin{array}{l}\text { Genus } 2 \text { HEC } C: Y^{2}=F(X), F=X^{5}+f_{3} X^{3}+f_{2} X^{2}+f_{1} X+f_{0} \\
\text { Reduced Divisors } D_{1}=\left(U_{1}, V_{1}\right), U_{1}=X+u_{10}, V_{1}=v_{10}\end{array}$ & \\
\hline Output & $\begin{array}{l}\text { Reduced Divisor } D_{3}=\left(U_{3}, V_{3}\right)=3 D_{1} \\
U_{3}=X^{2}+u_{31} X+u_{30}, V_{3}=v_{31} X+v_{30}\end{array}$ & \\
\hline Step & Expression & Cost \\
\hline 1 & $\begin{array}{l}\text { Compute } \boldsymbol{V}_{\mathbf{2}}=\boldsymbol{v}_{\mathbf{2 1}} \boldsymbol{X}+\boldsymbol{v}_{\mathbf{2 0}}(\text { See Equation (1)): } \\
\widetilde{u}_{10}=u_{10}^{2}, \widetilde{v}_{10}=v_{10}^{2}, t_{1}=5 \widetilde{u}_{10}, t_{2}=t_{1}+3 f_{3}, t_{3}=u_{10} t_{2} \\
t_{4}=t_{3}-2 f_{2}, t_{5}=u_{10} t_{4}, t_{6}=t_{5}+f_{1}, t_{7}=\left(2 v_{10}\right)^{-1} \\
v_{21}=t_{6} t_{7}, v_{20}=u_{10} v_{21}+v_{10}\end{array}$ & $1 I, 4 M, 2 S$ \\
\hline 2 & $\begin{array}{l}\text { Compute } d_{1}=\operatorname{gcd}\left(U_{1}, U_{2}\right)=X+u_{10}=e_{1} U_{1}+e_{2} U_{2}: \\
e_{1}=1, e_{2}=0 ;\end{array}$ & - \\
\hline 3 & $\begin{array}{l}\text { Compute } \boldsymbol{d}=\operatorname{gcd}\left(\boldsymbol{d}_{\mathbf{1}}, \boldsymbol{V}_{\mathbf{1}}+\boldsymbol{V}_{\mathbf{2}}\right)=\mathbf{1}=\boldsymbol{c}_{\mathbf{1}} \boldsymbol{d}_{\mathbf{1}}+\boldsymbol{c}_{\mathbf{2}}\left(\boldsymbol{V}_{\mathbf{1}}+\boldsymbol{V}_{\mathbf{2}}\right): \\
s_{1}=c_{1} e_{1}=c_{1}, s_{2}=c_{2} e_{2}=0, s_{3}=c_{2}=t_{7} ;\end{array}$ & - \\
\hline 4 & Compute $U^{\prime}=U_{1}^{3} d^{-2}=\left(X+u_{10}\right)^{3}$ : & - \\
\hline 5 & $\begin{array}{l}\text { Compute } \boldsymbol{V}^{\prime}=v_{\mathbf{2}}^{\prime} \boldsymbol{X}^{\mathbf{2}}+\boldsymbol{v}_{\mathbf{1}}^{\prime} \boldsymbol{X}+\boldsymbol{v}_{\mathbf{0}}^{\prime} \equiv\left[\boldsymbol{s}_{\mathbf{1}} U_{\mathbf{1}} V_{\mathbf{2}}+s_{\mathbf{3}}\left(\boldsymbol{V}_{\mathbf{1}} \boldsymbol{V}_{\mathbf{2}}+\boldsymbol{F}\right)\right] d^{-1} \bmod U^{\prime}: \\
\widetilde{v}_{21}=v_{21}^{2}, v_{2}^{\prime}=t_{7}\left(f_{2}-\widetilde{v}_{21}-u_{10}\left(t_{1}+t_{2}\right)\right) ; \\
v_{1}^{\prime}=v_{21}+2 u_{10} v_{2}^{\prime}, v_{0}^{\prime}=v_{20}+\widetilde{u}_{10} v_{2}^{\prime} ;\end{array}$ & $4 M, 1 S$ \\
\hline 6 & $\begin{array}{l}\text { Compute } \boldsymbol{U}_{\mathbf{3}}=\boldsymbol{X}^{\mathbf{2}}+\boldsymbol{u}_{\mathbf{3 1}} \boldsymbol{X}+\boldsymbol{u}_{\mathbf{3 0}}=\left(\boldsymbol{F}-\boldsymbol{V}^{\prime \mathbf{2}}\right) / \boldsymbol{U}^{\prime}: \\
u_{31}=-\left(v_{2}^{\prime} 2+3 u_{10}\right), u_{30}=f_{3}+t_{1}+\widetilde{u}_{10}+v_{2}^{\prime}\left(3 u_{10} v_{2}^{\prime}-2 v_{1}^{\prime}\right)\end{array}$ & $2 M, 1 S$ \\
\hline 7 & $\begin{array}{l}\text { Compute } \boldsymbol{V}_{\mathbf{3}}=\boldsymbol{v}_{31} \boldsymbol{X}+\boldsymbol{v}_{\mathbf{3 0}}=-\boldsymbol{V}^{\prime} \bmod \boldsymbol{U}_{\mathbf{3}}: \\
v_{31}=u_{31} v_{2}^{\prime}-v_{1}^{\prime}, v_{30}=u_{30} v_{2}^{\prime}-v_{0}^{\prime} ;\end{array}$ & $2 M$ \\
\hline Sum & & $1 I, 12 M, 4 S$ \\
\hline
\end{tabular}


Table 6. Explicit Formula for $D_{1}+D_{2}$ on a HEC of Genus 2 over $\mathbb{F}_{p}$ : $\mathrm{ADD}^{1+2 \rightarrow 2}$

\begin{tabular}{|c|c|c|}
\hline Input & $\begin{array}{l}\text { Genus } 2 \text { HEC } C: Y^{2}=F(X), F=X^{5}+f_{3} X^{3}+f_{2} X^{2}+f_{1} X+f_{0} \\
\text { Reduced Divisors } D_{1}=\left(U_{1}, V_{1}\right) \text { and } D_{2}=\left(U_{2}, V_{2}\right) \\
U_{1}=X+u_{10}, V_{1}=v_{10}, U_{2}=\left(X+u_{10}\right)\left(X+u_{20}\right), V_{2}=v_{21} X+v_{20}\end{array}$ & \\
\hline Output & $\begin{array}{l}\text { Reduced Divisor } D_{3}=\left(U_{3}, V_{3}\right)=D_{1}+D_{2} \\
U_{3}=X^{2}+u_{31} X+u_{30}, V_{3}=v_{31} X+v_{30}\end{array}$ & \\
\hline Step & Expression & Cost \\
\hline 1 & $\begin{array}{l}\text { Compute } \boldsymbol{V}_{\mathbf{2}}=\boldsymbol{v}_{\mathbf{2 1}} \boldsymbol{X}+\boldsymbol{v}_{\mathbf{2 0}}(\text { See Equation (2)): } \\
t_{1}=u_{10}-u_{20}, t_{2}=v_{20}-v_{10}, t_{3}=2 v_{10}, t_{4}=v_{20} u_{10}, t_{5}=v_{10} u_{20} \\
t_{6}=\left(t_{1} t_{3}\right)^{-1}, t_{7}=t_{3} t_{6}, t_{8}=t_{1} t_{6}, v_{21}=t_{2} t_{7}, v_{20}=\left(t_{4}-t_{5}\right) t_{7}\end{array}$ & $1 I, 7 M$ \\
\hline 2 & $\begin{array}{l}\text { Compute } d_{1}=\operatorname{gcd}\left(U_{1}, U_{2}\right)=X+u_{10}=e_{1} U_{1}+e_{2} U_{2}: \\
e_{1}=1, e_{2}=0\end{array}$ & - \\
\hline 3 & $\begin{array}{l}\text { Compute } \boldsymbol{d}=\operatorname{gcd}\left(\boldsymbol{d}_{\mathbf{1}}, \boldsymbol{V}_{\mathbf{1}}+\boldsymbol{V}_{\mathbf{2}}\right)=\mathbf{1}=\boldsymbol{c}_{\mathbf{1}} \boldsymbol{d}_{\mathbf{1}}+\boldsymbol{c}_{\mathbf{2}}\left(\boldsymbol{V}_{\mathbf{1}}+\boldsymbol{V}_{\mathbf{2}}\right): \\
s_{1}=c_{1} e_{1}=c_{1}, s_{2}=c_{2} e_{2}=0, s_{3}=c_{2}=t_{8}\end{array}$ & - \\
\hline 4 & Compute $U^{\prime}=U_{1} U_{2} d^{-2}=\left(X+u_{10}\right)^{2}\left(X+u_{20}\right)$ : & - \\
\hline 5 & $\begin{array}{l}\text { Compute } v^{\prime}=v_{2}^{\prime} X^{2}+v_{1}^{\prime} x+v_{0}^{\prime} \equiv\left[s_{1} U_{1} v_{2}+s_{3}\left(V_{1} V_{2}+F\right)\right] d^{-1} \bmod U^{\prime}: \\
\widetilde{u}_{10}=u_{10}^{2}, \widetilde{u}_{20}=u_{20}^{2}, \widetilde{v}_{21}=v_{21}^{2}, w_{1}=u_{10}+u_{20}, w_{2}=u_{10}+w_{1} \\
w_{3}=f_{2}-\widetilde{v}_{21}-w_{2}\left(f_{3}+\widetilde{u}_{10}+\widetilde{u}_{20}\right)-2 \widetilde{u}_{10} w_{1}, v_{2}^{\prime}=w_{3} t_{8} \\
v_{1}^{\prime}=v_{2}^{\prime} w_{1}+v_{21}, w_{4}=u_{10} u_{20}, v_{0}^{\prime}=v_{2}^{\prime} w_{4}+v_{20}\end{array}$ & $6 M, 3 S$ \\
\hline 6 & $\begin{array}{l}\text { Compute } \boldsymbol{U}_{\mathbf{3}}=\boldsymbol{X}^{\mathbf{2}}+\boldsymbol{u}_{\mathbf{3 1}} \boldsymbol{X}+\boldsymbol{u}_{\mathbf{3 0}}=\left(\boldsymbol{F}-\boldsymbol{V}^{\prime 2}\right) / \boldsymbol{U}^{\prime}: \\
u_{31}=-\left(v_{2}^{\prime 2}+w_{2}\right), w_{5}=u_{10}\left(w_{1}+u_{20}\right), u_{30}=f_{3}-2 v_{1}^{\prime} v_{2}^{\prime}-w_{5}\left(u_{31}+1\right)\end{array}$ & $3 M, 1 S$ \\
\hline 7 & $\begin{array}{l}\text { Compute } \boldsymbol{V}_{\mathbf{3}}=\boldsymbol{v}_{\mathbf{3 1}} \boldsymbol{X}+\boldsymbol{v}_{\mathbf{3 0}}=-\boldsymbol{V}^{\prime} \bmod \boldsymbol{U}_{\mathbf{3}}: \\
v_{31}=u_{31} v_{2}^{\prime}-v_{1}^{\prime}, v_{30}=u_{30} v_{2}^{\prime}-v_{0}^{\prime} ;\end{array}$ & $2 M$ \\
\hline Sum & & $1 I, 18 M, 4 S$ \\
\hline
\end{tabular}


Table 7. Explicit Formula for $2 D_{1}+D_{2}$ on a HEC of Genus 2 over $\mathbb{F}_{p}$ : $\mathrm{ADD}^{1+2 \rightarrow 2}$

\begin{tabular}{|c|c|c|}
\hline Input & $\begin{array}{l}\text { Genus } 2 \text { HEC } C: Y^{2}=F(X), F=X^{5}+f_{3} X^{3}+f_{2} X^{2}+f_{1} X+f_{0} \\
\text { Reduced Divisors } D_{1}=\left(U_{1}, V_{1}\right) \text { and } D_{2}=\left(U_{2}, V_{2}\right) \\
U_{1}=X+u_{10}, V_{1}=v_{10}, U_{2}=X^{2}+u_{21} X+u_{20}, V_{2}=v_{21} X+v_{20}\end{array}$ & \\
\hline Output & $\begin{array}{l}\text { Reduced Divisor } D_{3}=\left(U_{3}, V_{3}\right)=2 D_{1}+D_{2} \\
U_{3}=X^{2}+u_{31} X+u_{30}, V_{3}=v_{31} X+v_{30}\end{array}$ & \\
\hline Step & Expression & Cost \\
\hline 1 & $\begin{array}{l}\text { Compute the resultant } \boldsymbol{r} \text { of } \boldsymbol{U}_{\mathbf{1}} \text { and } \boldsymbol{U}_{\mathbf{2}} \text { : } \\
i_{0}=u_{10}-u_{21}, r=i_{0} u_{10}+u_{20}\end{array}$ & $1 M$ \\
\hline 2 & Compute the pseudo-inverse $I=-X+i_{0} \equiv r / U_{1} \bmod U_{2}$ : & - \\
\hline 3 & $\begin{array}{l}\text { Compute } \boldsymbol{S}^{\prime}=\boldsymbol{s}_{1}^{\prime} \boldsymbol{X}+\boldsymbol{s}_{\mathbf{0}}^{\prime}=\boldsymbol{r} \boldsymbol{S} \equiv\left(\boldsymbol{V}_{\mathbf{2}}-\boldsymbol{V}_{\mathbf{1}}\right) \boldsymbol{I} \bmod \boldsymbol{U}_{\mathbf{2}}: \\
w_{0}=v_{20}-v_{10}, s_{1}^{\prime}=u_{10} v_{21}-w_{0}, s_{0}^{\prime}=i_{0} w_{0}+u_{20} v_{21} ;\end{array}$ & $3 M$ \\
\hline 4 & Compute $S=s_{1} X+s_{0}=\left(s_{1}^{\prime} / r\right) X+\left(s_{0}^{\prime} / r\right):$ & - \\
\hline 5 & $\begin{array}{l}\text { Compute } \boldsymbol{U}^{\prime}=\left(\boldsymbol{k}-\boldsymbol{S}\left(\boldsymbol{l}+\mathbf{2} \boldsymbol{V}_{\mathbf{1}}\right)\right) / \boldsymbol{U}_{\mathbf{2}}=\boldsymbol{X}^{\mathbf{2}}+\boldsymbol{u}_{\mathbf{1}}^{\prime} \boldsymbol{X}+\boldsymbol{u}_{\mathbf{0}}^{\prime}: \\
R=r^{2}, w_{0}=u_{10}+u_{21}, w_{1}=f_{3}+u_{10}^{2}, u_{1}^{\prime}=-\left(s_{1}^{\prime 2}+R w_{0}\right) ; \\
u_{0}^{\prime}=R\left(w_{1}-u_{20}+u_{21} w_{0}\right)-s_{1}\left(s_{1} i_{0}+2 s_{0}\right) ;\end{array}$ & $5 M, 3 S$ \\
\hline 6 & $\begin{array}{l}\text { Compute the resultant } \tilde{\boldsymbol{r}} \text { of } \boldsymbol{U}_{\mathbf{1}} \text { and } \boldsymbol{U}^{\prime}: \\
\tilde{i}_{0}=R u_{10}-u_{1}^{\prime}, \tilde{r}=\tilde{i}_{0} u_{10}+u_{0}^{\prime} \\
\text { If } \tilde{r}=0 \text { then factor } U^{\prime}=\left(X+u_{10}\right)\left(X+u_{20}^{\prime}\right) \text { and see Table } \mathbf{6}\end{array}$ & $2 M$ \\
\hline 7 & Compute the pseudo-inverse $\tilde{I}=-X+\tilde{i}_{0} \equiv \tilde{r} / U_{1} \bmod U^{\prime}:$ & - \\
\hline 8 & $\begin{array}{l}\text { Compute } \tilde{\boldsymbol{S}}^{\prime}=\tilde{\boldsymbol{s}}_{1}^{\prime} \boldsymbol{X}+\tilde{\boldsymbol{s}}_{\mathbf{0}}^{\prime}=\tilde{\boldsymbol{r}} \tilde{\boldsymbol{S}} \equiv-\boldsymbol{S}^{\prime}-\mathbf{2} \boldsymbol{V}_{\mathbf{1}} \tilde{\boldsymbol{I}} \bmod \boldsymbol{U}^{\prime}: \\
\tilde{s}_{1}^{\prime}=2 v_{10}-s_{1}^{\prime}, \tilde{s}_{0}^{\prime}=-\left(R s_{0}^{\prime}+2 v_{10} \tilde{i}_{0}\right) ;\end{array}$ & $2 M$ \\
\hline 9 & 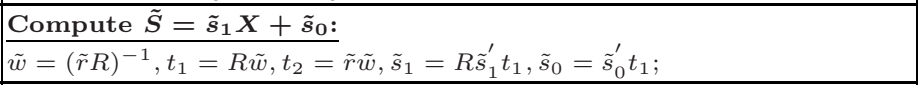 & $1 I, 6 M$ \\
\hline 10 & 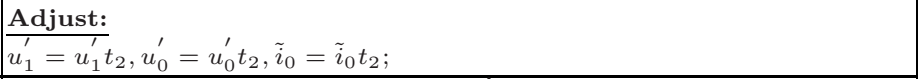 & $3 M$ \\
\hline 11 & $\begin{array}{l}\text { Compute } \boldsymbol{U}_{\mathbf{3}}=\left(\boldsymbol{k}-\tilde{\boldsymbol{S}}\left(\tilde{\boldsymbol{l}}+\mathbf{2} \boldsymbol{V}_{\mathbf{1}}\right)\right) / \boldsymbol{U}^{\prime}=\boldsymbol{X}^{\mathbf{2}}+\boldsymbol{u}_{\mathbf{3 1}} \boldsymbol{X}+\boldsymbol{u}_{\mathbf{3 0}}: \\
\tilde{w}_{0}=u_{10}+u_{1}^{\prime}, u_{31}=-\left(\tilde{s}_{1}^{2}+\tilde{w}_{0}\right), u_{30}=w_{1}-u_{0}^{\prime}+u_{1}^{\prime} \tilde{w}_{0}-\tilde{s}_{1}\left(\tilde{s}_{1} \tilde{i}_{0}+2 \tilde{s}_{0}\right)\end{array}$ & $3 M, 1 S$ \\
\hline 12 & $\begin{array}{l}\text { Compute } \boldsymbol{V}_{\mathbf{3}}=-\left(\tilde{\boldsymbol{l}}+\boldsymbol{V}_{\mathbf{1}}\right) \bmod \boldsymbol{U}_{\mathbf{3}}=\boldsymbol{v}_{\mathbf{3 1}} \boldsymbol{X}+\boldsymbol{v}_{\mathbf{3 0}}: \\
v_{31}=\tilde{s}_{1}\left(u_{31}-u_{10}\right)-\tilde{s}_{0}, v_{30}=\tilde{s}_{1} u_{30}-\tilde{s}_{0} u_{10}-v_{10}\end{array}$ & $3 M$ \\
\hline Sum & & $I, 28 M, 4 S$ \\
\hline
\end{tabular}


Table 8. Explicit Formula for $2 D_{1}+D_{2}$ on a HEC of Genus 2 over $\mathbb{F}_{p}$ : $\mathrm{ADD}^{2+1 \rightarrow 2}$

\begin{tabular}{|c|c|c|}
\hline Input & $\begin{array}{l}\text { Genus } 2 \text { HEC } C: Y^{2}=F(X), F=X^{5}+f_{3} X^{3}+f_{2} X^{2}+f_{1} X+f_{0} \\
\text { Reduced Divisors } D_{1}=\left(U_{1}, V_{1}\right) \text { and } D_{2}=\left(U_{2}, V_{2}\right) \\
U_{1}=X^{2}+u_{11} X+u_{10}, V_{1}=v_{11} X+v_{10}, U_{2}=X+u_{20}, V_{2}=v_{20}\end{array}$ & \\
\hline Output & $\begin{array}{l}\text { Reduced Divisor } D_{3}=\left(U_{3}, V_{3}\right)=2 D_{1}+D_{2} \\
U_{3}=X^{2}+u_{31} X+u_{30}, V_{3}=v_{31} X+v_{30}\end{array}$ & \\
\hline Step & Expression & Cost \\
\hline 1 & $\begin{array}{l}\text { Compute the resultant } \boldsymbol{r}=\boldsymbol{U}_{\mathbf{1}} \bmod \boldsymbol{U}_{\mathbf{2}}: \\
r=u_{10}-\left(u_{11}-u_{20}\right) u_{20} ;\end{array}$ & $1 M$ \\
\hline 2 & Compute the inverse $i \equiv 1 / U_{1} \bmod U_{2}$ : & - \\
\hline 3 & $\begin{array}{l}\text { Compute } \boldsymbol{S}=\boldsymbol{s}_{0} \equiv\left(\boldsymbol{V}_{\mathbf{2}}-\boldsymbol{V}_{\mathbf{1}}\right) \boldsymbol{i} \bmod \boldsymbol{U}_{\mathbf{2}}: \\
s_{0}=v_{20}-v_{10}-v_{11} u_{20}\end{array}$ & $1 M$ \\
\hline 4 & $\begin{array}{l}\text { Compute } K=\left(\boldsymbol{F}-\boldsymbol{V}_{1}^{2}\right) / U_{1}=\boldsymbol{X}^{3}+\boldsymbol{k}_{2} \boldsymbol{X}^{2}+\boldsymbol{k}_{1} \boldsymbol{X}+\boldsymbol{k}_{0}: \\
k_{1}=f_{3}+u_{11}^{2}-u_{10}\end{array}$ & $1 S$ \\
\hline 5 & $\begin{array}{l}\text { Compute } \boldsymbol{U}^{\prime}=\left(\boldsymbol{k}-\boldsymbol{S}\left(\boldsymbol{l}+\mathbf{2} \boldsymbol{V}_{\mathbf{1}}\right)\right) / \boldsymbol{U}_{\mathbf{2}}=\boldsymbol{X}^{\mathbf{2}}+\boldsymbol{u}_{\mathbf{1}}^{\prime} \boldsymbol{X}+\boldsymbol{u}_{\mathbf{0}}^{\prime}: \\
R=r^{2}, u_{1}^{\prime}=-\left(s_{0}^{2}+R\left(u_{11}+u_{20}\right)\right), u_{0}^{\prime}=R k_{1}-s_{0}\left(s_{0} u_{11}+2 r v_{11}\right)-u_{20} u_{1}^{\prime} ;\end{array}$ & $6 M, 2 S$ \\
\hline 6 & $\begin{array}{l}\text { Compute the resultant } \tilde{\boldsymbol{r}} \text { of } \boldsymbol{U}_{1} \text { and } \boldsymbol{U}^{\prime} \text { : } \\
\tilde{\tilde{i}}_{1}=u_{1}^{\prime}-R u_{11}, \tilde{w}=R u_{10}-u_{0}^{\prime}, \tilde{i}_{0}=\tilde{i}_{1} u_{1}^{\prime}+R \tilde{w}, \tilde{r}=\tilde{i}_{0} \tilde{w}+\tilde{i}_{1}^{2} u_{0}^{\prime} \\
\text { If } \tilde{r}=0 \text { then see Table }\end{array}$ & $6 M, 1 S$ \\
\hline 7 & Compute the pseudo-inverse $\tilde{I}=\tilde{i}_{1} X+\tilde{i}_{0} \equiv \tilde{r} / U_{1} \bmod U^{\prime}:$ & - \\
\hline 8 & $\begin{array}{l}\text { Compute } \tilde{\boldsymbol{S}}^{\prime}=\tilde{s}_{1}^{\prime} \boldsymbol{X}+\tilde{s}_{0}^{\prime}=\tilde{r} \tilde{\boldsymbol{S}} \equiv-\boldsymbol{S}-\mathbf{2} \boldsymbol{V}_{\mathbf{1}} \tilde{\boldsymbol{I}} \mathbf{m o d} \boldsymbol{U}^{\prime}: \\
\tilde{w}_{0}=\tilde{i}_{0} v_{10}, \tilde{w}_{1}=\tilde{i}_{1} v_{11}, \tilde{s}_{0}^{\prime}=-\left(R r s_{0}+2\left(\tilde{w}_{0}-u_{0}^{\prime} \tilde{w}_{1}\right)\right) ; \\
\tilde{s}_{1}^{\prime}=-2\left(\left(\tilde{i}_{0}+R \tilde{i}_{1}\right)\left(v_{10}+v_{11}\right)-\tilde{w}_{0}-\tilde{w}_{1}\left(R+u_{1}^{\prime}\right)\right) ; \text { If } \tilde{s}_{1}^{\prime}=0 \text { See Below }\end{array}$ & $8 M$ \\
\hline 9 & $\begin{array}{l}\text { Compute } \tilde{\boldsymbol{S}}^{\prime \prime}=\boldsymbol{X}+\tilde{s}_{0} / \tilde{s}_{1}=\boldsymbol{X}+\tilde{s}_{0}^{\prime} / \tilde{s}_{1}^{\prime} \text { and } \tilde{s}_{1}: \\
\tilde{w}_{0}=\tilde{r} \tilde{s}_{1}^{\prime}, \tilde{w}_{1}=\left(R \tilde{w}_{0}\right)^{-1}, R_{1}=\tilde{w}_{0} \tilde{w}_{1}, R_{2}=R_{1}^{2}, R_{3}=R_{1} R_{2}, \tilde{w}_{2}=\tilde{r} \tilde{w}_{1} R_{2} ; \\
\tilde{w}_{3}=\tilde{s}_{1}^{\prime}{ }_{1}^{2} \tilde{w}_{1} R_{3}, \tilde{w}_{4}=\tilde{r} \tilde{w}_{2} R_{3}, \tilde{w}_{5}=\tilde{w}_{4}^{2}, \tilde{s}_{0}^{\prime \prime}=\tilde{s}_{0}^{\prime} \tilde{w}_{2} R_{2} ;\end{array}$ & $1 I, 12 M, 3 S$ \\
\hline 10 & $\begin{array}{l}\text { Adjust: } \\
u_{1}^{\prime}=u_{1}^{\prime}\end{array} R_{1}, u_{0}^{\prime}=u_{0}^{\prime} R_{1}, \tilde{i}_{1}=\tilde{i}_{1} R_{1}$ & $3 M$ \\
\hline 11 & $\begin{array}{l}\text { Compute } \tilde{l}^{\prime}=\tilde{S}^{\prime \prime} u_{1}=X^{3}+\tilde{l}_{2}^{\prime} \boldsymbol{X}^{2}+\tilde{l}_{1}^{\prime} \boldsymbol{X}+\tilde{l}_{0}^{\prime}: \\
\tilde{l}_{2}^{\prime}=u_{11}+\tilde{s}_{0}^{\prime \prime}, \tilde{l}_{1}^{\prime}=u_{11} \tilde{s}_{0}^{\prime \prime}+u_{10}, \tilde{l}_{0}^{\prime}=u_{10} \tilde{s}_{0}^{\prime \prime}\end{array}$ & $2 M$ \\
\hline 12 & $\begin{array}{l}\text { Compute } U_{3}=\left(\tilde{s}\left(\tilde{l}+2 V_{1}\right)-\boldsymbol{k}\right) / U^{\prime}=\boldsymbol{X}^{2}+u_{31} \boldsymbol{X}+u_{30}: \\
u_{30}=\left(\tilde{s}_{0}^{\prime \prime}-u_{1}^{\prime}\right)\left(\tilde{s}_{0}^{\prime \prime}-\tilde{i}_{1}\right)-u_{0}^{\prime}+\tilde{l}_{1}^{\prime}+2 v_{11} \tilde{w}_{4}+\left(u_{1}^{\prime}+u_{11}\right) \tilde{w}_{5} ; \\
u_{31}=2 \tilde{s}_{0}^{\prime \prime}-\tilde{i}_{1}-\tilde{w}_{5} ;\end{array}$ & $3 M$ \\
\hline 13 & $\begin{array}{l}\text { Compute } \boldsymbol{V}_{\mathbf{3}}=-\left(\tilde{\boldsymbol{l}}+\boldsymbol{V}_{\mathbf{1}}\right) \bmod \boldsymbol{U}_{\mathbf{3}}=\boldsymbol{v}_{\mathbf{3 1}} \boldsymbol{X}+\boldsymbol{v}_{\mathbf{3 0}}: \\
w_{1}=\tilde{l}_{2}^{\prime}-u_{31}, w_{2}=u_{31} w_{1}+u_{30}-\tilde{l}_{1}^{\prime}, v_{31}=w_{2} \tilde{w}_{3}-v_{11} \\
w_{2}=u_{30} w_{1}-\tilde{l}_{0}^{\prime}, v_{30}=w_{2} \tilde{w}_{3}-v_{10}\end{array}$ & $4 M$ \\
\hline Sum & $\tilde{s}_{1}^{\prime} \neq 0$ & $I, 46 M, 7 S$ \\
\hline 9 ' & $\begin{array}{l}\text { Compute } \tilde{s}_{0}: \\
\tilde{w}_{1}=(\tilde{r} R)^{-1}, t_{1}=\tilde{r} \tilde{w}_{1}, t_{2}=R \tilde{w}_{1}, \tilde{s}_{0}=R \tilde{s}_{0}^{\prime} t_{2} ;\end{array}$ & $\overline{1 I, 5 M}$ \\
\hline $10^{\prime}$ & $\begin{array}{l}\text { Adjust: } \\
u_{1}^{\prime}=u_{1}^{\prime} t_{1}, u_{0}^{\prime}=u_{0}^{\prime} t_{1} ;\end{array}$ & $2 M$ \\
\hline $11^{\prime}$ & $\begin{array}{l}\text { Compute } U_{3}=\left(\boldsymbol{k}-\tilde{s}\left(\tilde{l}+2 V_{1}\right)\right) / U^{\prime}=X+u_{30}: \\
u_{30}=-\left(u_{1}^{\prime}+u_{11}+\tilde{s}_{0}^{2}\right) ;\end{array}$ & $1 S$ \\
\hline $12^{\prime}$ & $\begin{array}{l}\text { Compute } \boldsymbol{V}_{\mathbf{3}}=-\left(\tilde{\boldsymbol{l}}+\boldsymbol{V}_{\mathbf{1}}\right) \bmod \boldsymbol{U}_{\mathbf{3}}=\boldsymbol{v}_{\mathbf{3 0}}: \\
w_{1}=\tilde{s}_{0}\left(u_{1}^{\prime}+u_{30}\right)+v_{11}, w_{2}=\tilde{s}_{0}+v_{10}, v_{30}=u_{0}^{\prime} w_{1}-w_{2} ;\end{array}$ & $2 M$ \\
\hline Sum & $\tilde{s}_{1}^{\prime}=0$ & $I, 31 M, 5 S$ \\
\hline
\end{tabular}


Table 9. Explicit Formula for $D_{1}+D_{2}$ on a HEC of Genus 2 over $\mathbb{F}_{p}$ : $\mathrm{ADD}^{2+2 \rightarrow 2}$

\begin{tabular}{|c|c|c|}
\hline Input & $\begin{array}{l}\text { Genus } 2 \text { HEC } C: Y^{2}=F(X), F=X^{5}+f_{3} X^{3}+f_{2} X^{2}+f_{1} X+f_{0} \\
\text { Reduced Divisors } D_{1}=\left(U_{1}, V_{1}\right) \text { and } D_{2}=\left(U_{2}, V_{2}\right) \\
U_{1}=X^{2}+u_{11} X+u_{10}=\left(X+u_{p 1}\right)\left(X+u_{p 2}\right), V_{1}=v_{11} X+v_{10} \\
U_{2}=X^{2}+u_{21} X+u_{20}=\left(X+u_{p 1}\right)\left(X+u_{p 3}\right), V_{2}=v_{21} X+v_{20}\end{array}$ & \\
\hline Output & $\begin{array}{l}\text { Reduced Divisor } D_{3}=\left(U_{3}, V_{3}\right)=D_{1}+D_{2} \\
U_{3}=X^{2}+u_{31} X+u_{30}, V_{3}=v_{31} X+v_{30}\end{array}$ & \\
\hline Step & Expression & Cost \\
\hline 1 & $\begin{array}{l}\text { Compute } d_{1}=\operatorname{gcd}\left(U_{1}, U_{2}\right)=X+u_{p 1}=e_{1} U_{1}+e_{2} U_{2}: \\
e_{1}=1, e_{2}=0\end{array}$ & - \\
\hline 2 & $\begin{array}{l}\text { Compute } d=\operatorname{gcd}\left(d_{1}, V_{1}+V_{2}\right)=c_{1} d_{1}+c_{2}\left(V_{1}+V_{2}\right): \\
\text { If } d=X+u_{10} \text { then see below, else } d=1 \text { and we have } \\
s_{1}=c_{1} e_{1}=c_{1}, s_{2}=c_{2} e_{2}=0, s_{3}=c_{2}\end{array}$ & - \\
\hline 3 & $\begin{array}{l}\text { Compute } \boldsymbol{U}^{\prime}=\boldsymbol{U}_{\mathbf{1}} \boldsymbol{U}_{\mathbf{2}} \boldsymbol{d}^{-\mathbf{2}}=\boldsymbol{X}^{\mathbf{4}}+\boldsymbol{u}_{\mathbf{3}}^{\prime} \boldsymbol{X}^{\mathbf{3}}+\boldsymbol{u}_{\mathbf{2}}^{\prime} \boldsymbol{X}^{\mathbf{2}}+\boldsymbol{u}_{\mathbf{1}}^{\prime} \boldsymbol{X}+\boldsymbol{u}_{0}^{\prime}: \\
u_{3}^{\prime}=u_{11}+u_{21}, t_{0}=u_{11} u_{21}, u_{2}^{\prime}=u_{10}+u_{20}+t_{0}, u_{0}^{\prime}=u_{10} u_{20} ; \\
u_{1}^{\prime}=\left(u_{11}+u_{10}\right)\left(u_{20}+u_{21}\right)-t_{0}-u_{0}^{\prime} ;\end{array}$ & $3 M$ \\
\hline 4 & $\begin{array}{l}\text { Compute } v^{\prime}=v_{3}^{\prime} X^{3}+v_{2}^{\prime} X^{2}+v_{1}^{\prime} X+v_{0}^{\prime} \equiv\left[s_{1} U_{1} V_{2}+s_{3}\left(V_{1} v_{2}+F\right)\right] d^{-1} \bmod U^{\prime}: \\
t_{1}=v_{11}+v_{21}, t_{2}=u_{11} v_{21}, t_{3}=u_{21} v_{20}, t_{4}=v_{11} v_{21}, t_{5}=v_{10} v_{20} ; \\
t_{6}=\left(v_{10}+v_{11}\right)\left(v_{20}+v_{21}\right)-t_{4}-t_{5}, t_{7}=\left(u_{11}+u_{21}\right)\left(v_{20}+v_{21}\right)-t_{2}-t_{3} ; \\
t_{8}=v_{10}+v_{20}-t_{1} u_{p 1}, t_{9}=f_{3}-t_{1} v_{21}-u_{2}^{\prime}+u_{3}^{\prime 2}, t_{10}=\left(t_{8} t_{9}\right)^{-1} ; \\
c_{2}=t_{9} t_{10}, v_{3}^{\prime}=c_{2} t_{9}, v_{2}^{\prime}=c_{2}\left(f_{2}+t_{4}-t_{1}\left(t_{2}+v_{20}\right)-u_{1}^{\prime}+u_{2}^{\prime} u_{3}^{\prime}\right) ; \\
v_{1}^{\prime}=c_{2}\left(f_{1}+t_{6}-t_{1} t_{7}-u_{0}^{\prime}+u_{1}^{\prime} u_{3}^{\prime}\right), v_{0}^{\prime}=c_{2}\left(f_{0}+t_{5}-t_{1} t_{3}+u_{0}^{\prime} u_{3}^{\prime}\right) ;\end{array}$ & $1 I, 20 M, 1 S$ \\
\hline 5 & $\begin{array}{l}\text { Compute } \boldsymbol{U}_{3}=\boldsymbol{X}^{\mathbf{2}}+\boldsymbol{u}_{\mathbf{3 1}} \boldsymbol{X}+\boldsymbol{u}_{\mathbf{3 0}}=\left(\boldsymbol{V}^{\prime 2}-\boldsymbol{F}\right) / \boldsymbol{U}^{\prime}: \\
t_{1}=t_{8}^{2} t_{10}, u_{31}=t_{1}\left(2 v_{2}^{\prime}-t_{1}\right)-u_{3}^{\prime}, u_{30}=\left(v_{2}^{\prime} t_{1}\right)^{2}+2 v_{1}^{\prime} t_{1}-u_{2}^{\prime}-u_{3}^{\prime} u_{31}\end{array}$ & $5 M, 2 S$ \\
\hline 6 & $\begin{array}{l}\text { Compute } \boldsymbol{V}_{\mathbf{3}}=\boldsymbol{v}_{\mathbf{3 1}} \boldsymbol{X}+\boldsymbol{v}_{\mathbf{3 0}}=-\boldsymbol{V}^{\prime} \bmod \boldsymbol{U}_{\mathbf{3}}: \\
t_{2}=u_{31} v_{3}^{\prime}-v_{2}^{\prime}, v_{31}=u_{30} v_{3}^{\prime}-v_{1}^{\prime}-u_{31} t_{2}, v_{30}=-\left(u_{30} t_{2}+v_{0}^{\prime}\right) ;\end{array}$ & $4 M$ \\
\hline Sum & $d=1$ & $1 I, 32 M, 3 S$ \\
\hline 3 ' & $\begin{array}{l}\text { Compute } \boldsymbol{U}_{\mathbf{3}}=\boldsymbol{U}_{\mathbf{1}} \boldsymbol{U}_{\mathbf{2}} \boldsymbol{d}^{-\mathbf{2}}=\left(\boldsymbol{X}+\boldsymbol{u}_{p \mathbf{2}}\right)\left(\boldsymbol{X}+\boldsymbol{u}_{\boldsymbol{p} \mathbf{3}}\right): \\
u_{31}=u_{p 2}+u_{p 3}, u_{30}=u_{p 2} u_{p 3}\end{array}$ & $1 M$ \\
\hline $4^{\prime}$ & $\begin{array}{l}\text { Compute } \boldsymbol{V}^{\prime}=\boldsymbol{v}_{\mathbf{3 1}} \boldsymbol{X}+\boldsymbol{v}_{\mathbf{3 0}}: \\
t_{0}=\left(u_{p 2}-u_{p 3}\right)^{-1}, t_{1}=v_{11} u_{p 2}+v_{10}, t_{2}=v_{21} u_{p 3}+v_{20} \\
t_{3}=t_{2}-t_{1}, v_{31}=t_{0} t_{3}, t_{4}=t_{2} u_{p 2}-t_{1} u_{p 3}, v_{30}=t_{0} t_{4}\end{array}$ & $1 I, 6 M$ \\
\hline Sum & $d=X+u_{10}$ & $I, 7 M$ \\
\hline
\end{tabular}

